(C2018, Elsevier. Licensed under the Creative Commons Attribution-NonCommercialNoDerivatives 4.0 International http://creativecommons.org/about/downloads

cc) $(1) \Theta$ 


\title{
Precision Landing Using an Adaptive Fuzzy Multi-Sensor Data Fusion Architecture
}

\author{
Mohammad Al-Sharman, Bara J. Emran, Mohammad A. Jaradat, Homayoun Najjaran, Raghad Al-Husari, \\ and Yahya Zweiri
}

\begin{abstract}
The positional inaccuracies associated with the GPS/INS measurements make the terminal phase of the normal GPS/INS landing system imprecise. To solve this problem, an adaptive fuzzy data fusion algorithm is developed to obtain more accurate state estimates while the vehicle approaches the landing surface. This algorithm takes the translational displacements in $x$ and $y$ from the mounted Optical Flow (OF) sensor and fuses them with the INS attitude measurements and the altimeter measurements. This low cost adaptive algorithm can be used for vertical landings in areas where GPS outages might happen or in GPS denied areas. The adaptation is governed by imposing appropriate assumptions under which the filter measurement noise matrix $R$ is predicted. The $R$ matrix is continuously adjusted through a fuzzy inference system (FIS) based on the Kalman innovative sequence and the applied covariance-matching technique. This adaptive fuzzy Kalman fusion algorithm (AFKF) is used to estimate the vehicle's states while landing is being commanded. AFKF results are compared with these obtained using a classical Kalman estimation technique. The AFKF algorithm shows better states estimates than its conventional counterpart does. Compared to prior landing systems, the proposed low cost AFKF has achieved a precision landing with less than $10 \mathrm{~cm}$ maximum estimated position error. Real precision landing flights were conducted to demonstrate the validity of the proposed intelligent estimation method.
\end{abstract}

Index Terms-Precision Landing; Quadrotor, Intelligent Adaptive Estimation; Sensor Fusion; Optical Flow Sensor; Sensor Modeling.

\section{INTRODUCTION}

A successful utilization of unmanned aerial Vertical Takeoff and Landing (VTOL) vehicles in missions that require a high degree of autonomy necessitates accurate and fast updated measurements from the onboard navigational sensors. For exa-

Mohammad Al-Sharman is a Research Associate, Robotics Institute, Khalifa University of Science and Technology, P.O. Box 127788, Abu Dhabi, UAE (email:alsharman.mohammad@gmail.com).

Bara J. Emran is with the Department of Mechanical Engineering, University of British Columbia, Kelowna, Canada (e-mail: Bara.Emran@ubc.ca).

M. A. Jaradat is with the Department of Mechanical Engineering, the American University of Sharjah, Sharjah, United Arab Emirates (mjaradat@ aus.edu)/ Jordan University of Science \& technology, Irbid, Jordan. Homayoun Najjaran is with the Department of Mechanical Engineering, University of British Columbia, Kelowna, Canada (e-mail: h.najjaran@ubc.ca). Raghad Al husari is with the Department of Electrical Engineering, Khalifa University, Abu Dhabi, UAE (e-mail: raghad.husari@kustar.ac.ae).

Yahya Zweiri is with the Faculty of Science, Engineering and Computing, Kingston University London, London SW15 3DW, UK and a Visiting Associate Professor, Robotics Institute, Khalifa University of Science and Technology, P.O. Box 127788, Abu Dhabi, United Arab Emirates (e-mail: y.zweiri@kingston.ac.uk). -mple, the quality of the Global Positioning System (GPS) becomes low when the Unmanned Aerial Vehicle (UAV) is approaching the ground, and a landing with few meters error might result. Therefore, the use of other more precise sensors is needed for the development of the sensor fusion design $[1,2,3$, 4].

Vision sensors have been employed in designing autonomous landing systems in recent years due to their precision and high update rate measurements [5, 6]. A vision-based helicopterlanding algorithm was proposed in [7]. The study achieved a precise landing with an average position error equal to $47 \mathrm{~cm}$. However, their solution is computationally heavy and ill-suited for vehicles that have smaller payloads. In [8], a pattern of InfraRed (IR) LEDs organized in a T-shape and a Wii camera was utilized to perform an indoor auto takeoff, hovering and landing. The algorithm performs well at $60 \mathrm{~cm}$ height; however, at higher altitudes, the positional error will be large and inaccurate TOL might occur. In [9], a vision off-the-shelf hardware was used to provide real time estimates of the UAV orientation and the position relative to the landing position. Similarly, [10] studies the 6 Degree of Freedom (DOF) pose estimation of a Miniature Air Vehicle (MAV) using on-board monocular vision solutions.

OF sensors are considered robust low cost navigational sensors for UAVs applications [11]. OF sensors are used to avoid collision, measure the altitude and for position stabilization during the landing stage. Furthermore, OF sensors are used for height estimation and terrain navigation [12]. In [13], the OF measurements were used to control the vertical landing on a non-stationary platform. Whereas, In [14], an OF sensor was utilized for the position estimation of a quadrotor, and an auto-landing with $30 \mathrm{~cm}$ position error was performed. In [15], PX4FLOW optical sensor was used to perform hovering in an outdoor flight trajectory for Cheetah quadrotor. The optical flow components measured by the PX4FLOW sensor are compensated for the $3 \mathrm{D}$ rotations and transformed to the metric scale.

Precise state estimation is needed for the UAV to perform successful autonomous flight. However, obtaining accurate states estimate is challenging due to the sensor drifts, noise on the onboard Commercial off-the-shelf (COTS) sensors and measurement bias [16]. Low cost COTS sensors with such expected errors are usually utilized in UAVs because of their lightweight, low power consumption and compact size. By fusing the measurements of different precise sensors, the chance of obtaining accurate estimates would be definitely higher. For example, in [17], the readings of the kinematic OF model are fused with the measurements of the GPS/INS to estimate the 3D velocity states and position of an object. In $[18,19,16]$, a high- 
accuracy helicopter's attitude and flapping states estimation was addressed using the Kalman filter. The unmeasured flapping angles of the Maxi Joker 3 helicopter were estimated with maximum error not exceeding $0.3^{\circ}$.

The accuracy of the estimation algorithm in the Kalman filter is linked with the accuracy of the a priori information of the process and measurement noise statistics which are represented by the $R$ and $Q$ matrices [20,21]. Practically, inaccurate priori information will degrade the performance of the estimator, and a divergence of the filter might result. Therefore, the adaptive Kalman filter has been devised to tackle the problem of having imperfect a priori information [22, 23, 24]. The Kalman filter can be adapted using different procedures, i.e., Innovativebased Adaptive Estimation (IAE) and Multiple Model Adaptive Estimation (MMAE) [20]. The IAE technique depends on the enhancement of the filter performance via the adaptation of the matrices $R$ and $Q$ which are based on the filter innovation sequence. In [21, 25, 26], the IAE adaptation approach proves its capability of working with unknown measurement noise characteristics in the Kalman filter. Moreover, applying the fuzzy logic rules to adjust the statistical matrices has been studied in a number of published research papers. The fuzzyadapted Kalman filter shows better performance in rejecting the measurement noise and estimating the navigational states accurately [27, 28, 29].

In this paper, the problem of precision terminal landing phase has been tackled using intelligent adaptive low cost multisensor data fusion architecture. This architecture proposes a novel multi-sensor data fusion between the experimentally obtained OF sensors' model, altimeter and INS solution for vertical precision landing applications. Compared to prior landing systems, the proposed integrated solution has succeeded in performing an autonomous precision landing in GPS denied environments with less than $5 \mathrm{~cm}$ estimated altitude error. Moreover, the proposed intelligent estimation technique has shown high degree of robustness in the presence of external disturbances compared to the normal estimation techniques.

The following sections of the paper are organized as follows. Section II describes the quadrotor model used in this study and the optical flow modeling design. Section III represents the design of the proposed sensor fusion algorithm architecture. Simulation results are presented in Section IV while Section V demonstrates the experimental validation. Finally, Section VI concludes the paper.

\section{QUADROTOR MODEL}

Quadrotor has been increasingly studied as a preferred UAV platform for various applications. It is sustained in the air by the lift of four actuators, and it has six degrees of freedom. A typical quadrotor incorporated in multi-rotor cross platform is composed of four symmetrical arms. Each of its four actuators is connected to a propeller with fixed-pitch blade, and the axes of rotation of the four propellers are fixed and parallel to each other (see Figure 1). The system state variables can be controlled using different movements directly related to the propellers velocities, which allow the quadrotor to reach a desired altitude and attitude [30] .

\section{A. Reference frame}

This section describes the various reference frames and rotation matrix that are used to describe the position and the orientation of the quadrotor. In addition, it shows the nonlinear dynamic equation of the quadrotor. The linear position $(\Gamma)$ is determined using the vector between the origins of the B-frame and E-frame. The attitude of the vehicle is represented by the Euler angles $\left(\Theta=\left[\begin{array}{lll}\phi & \theta & \psi\end{array}\right]^{T}\right)$. These angles are defined by the orientation of B-frame with respect to the E-frame.

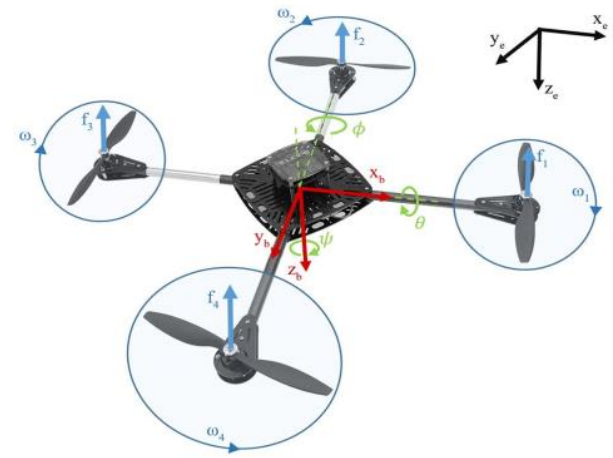

Figure 1: Quadrotor system

To map the orientation of a vector from B-frame to E-frame, a rotation matrix is needed [31]. This rotation matrix is given by:

$$
R=\left[\begin{array}{ccc}
c_{\theta} c_{\psi} & -c_{\phi} s_{\psi}+c_{\psi} s_{\phi} s_{\theta} & s_{\phi} s_{\psi}+c_{\phi} c_{\psi} s_{\theta} \\
c_{\theta} s_{\psi} & c_{\phi} c_{\psi}+s_{\phi} s_{\theta} s_{\psi} & -c_{\psi} s_{\phi}+c_{\phi} s_{\theta} s_{\psi} \\
-s_{\theta} & c_{\phi} s_{\theta} & c_{\phi} c_{\theta}
\end{array}\right]
$$

Where $c_{x}=\cos (x)$ and $\mathrm{s}_{x}=\sin (x)$.

Along with the rotation matrix, a transfer matrix is needed to map the relation between the angular velocity $(\boldsymbol{\omega})$ in the Bframe and Euler angles rates $(\dot{\boldsymbol{\Theta}})$ in the E-frame. This matrix is defined as follows [31]:

$$
\begin{aligned}
& \dot{\Theta}=T \omega \\
& \boldsymbol{T}=\left[\begin{array}{ccc}
1 & s_{\phi} t_{\theta} & c_{\phi} t_{\theta} \\
0 & c_{\phi} & -s_{\phi} \\
0 & s_{\phi} / c_{\theta} & c_{\phi} / c_{\theta}
\end{array}\right]
\end{aligned}
$$

Where $t_{x}=\tan (x)$.

\section{B. Dynamical Model}

Several dynamical models can be used to characterize the quadrotor dynamics. These models differ due to the various assumptions and simplifications that can be made to reduce the model complexities. As an illustration, having the vehicle aerodynamics into consideration would complicate the dynamical model to a high extent. Another well-used simplification is to consider the small angle assumption for miniature quadrotors. Reference [32] reviews dynamic models and controls of the quadrotor. A typical dynamical model of the quadrotor in E-frame could be derived using Newton's second law as follows: 


$$
\begin{gathered}
{\left[\begin{array}{c}
\ddot{x} \\
\ddot{y} \\
\ddot{z}
\end{array}\right]=\frac{1}{m}\left[\begin{array}{c}
s_{\psi} s_{\phi}+c_{\psi} s_{\theta} c_{\phi} \\
-c_{\psi} s_{\phi}+s_{\psi} s_{\theta} c_{\phi} \\
c_{\theta} c_{\phi}
\end{array}\right] T_{z}-\left[\begin{array}{l}
0 \\
0 \\
g
\end{array}\right]} \\
{\left[\begin{array}{c}
\ddot{\phi} \\
\ddot{\theta} \\
\ddot{\psi}
\end{array}\right]=\left[\begin{array}{c}
\left(\frac{I_{y}-I_{z}}{I_{x}}\right) \dot{\theta} \dot{\psi} \\
\left(\frac{I_{z}-I_{y}}{I_{y}}\right) \dot{\phi} \dot{\psi} \\
\left(\frac{I_{x}-I_{y}}{I_{z}}\right) \dot{\phi} \dot{\theta}
\end{array}\right]+\left[\begin{array}{c}
\frac{J_{p}}{I_{x}} \\
\frac{J_{p}}{I_{y}} \dot{\phi} \\
0
\end{array}\right] \Omega\left[\begin{array}{ccc}
\frac{1}{I_{x}} & 0 & 0 \\
0 & \frac{1}{I_{y}} & 0 \\
0 & 0 & \frac{1}{I_{z}}
\end{array}\right]\left[\begin{array}{c}
\tau_{\phi} \\
\tau_{\theta} \\
\tau_{\psi}
\end{array}\right]}
\end{gathered}
$$

where $\ddot{x}, \ddot{y}$ and $\ddot{z}$ represent the linear acceleration in $x, y$ and $z$ axes in E-frame, respectively. $m$ is the quadrotor's mass, and $g$ is the gravitational acceleration acting along the z-axis with respect to E-frame. $\ddot{\phi}, \ddot{\theta}$ and $\ddot{\psi}$ represent the angular acceleration around $x, y$ and $z$ axes, respectively, with respect to E-frame. $J_{P}$ denotes the total rotational moment of inertia around the propeller axis. Equation 5 defines the inputs of the quadrotor system, which represent the thrust force, roll torque, pitch torque and yaw torque, respectively.

$$
\begin{gathered}
{\left[\begin{array}{c}
T_{z} \\
\tau_{\phi} \\
\tau_{\theta} \\
\tau_{\psi}
\end{array}\right]=\left[\begin{array}{rrrr}
1 & 1 & 1 & 1 \\
-l & 0 & l & 0 \\
0 & l & 0 & -l \\
d & -d & d & -d
\end{array}\right]\left[\begin{array}{l}
f_{1} \\
f_{2} \\
f_{3} \\
f_{4}
\end{array}\right]} \\
f_{i}=k \cdot \omega_{i}^{2} \\
\Omega=-\omega_{1}+\omega_{2}-\omega_{3}+\omega_{4}
\end{gathered}
$$

Where $l$ is the quadrotor's arm length, $d, k$ and $\Omega_{i}$ represent the thrust factor, the drag factor and propellers speed respectively.

\section{Control Development}

This section describes the control system that is used to control the quadrotor through the simulation tests. Like any highly nonlinear system, quadrotors suffer from different constrains that compromise the quadrotor controller performance. Those constraints are not limited to strong subsystems coupling, model uncertainties, measurement noise and nonlinear dynamics. The underactuation problem is considered the main constraint that the quadrotors suffer from. In other words, the system cannot achieve instant acceleration in all arbitrary directions of its configuration space. Studies [33] and [34] show a comprehensive introduction on (VTOL) vehicles with basic control design ideas and principles. These articles explain system modelling of a general VTOL vehicle and consider different closed-loop control algorithms.

In this work, the researchers are interested in using a simple, yet sufficient, controller that achieves trajectory tracking for the desired command signal $\left[x_{\mathrm{c}}, y_{\mathrm{c}}, z_{\mathrm{c}}, \psi_{\mathrm{c}}\right]$; a more advanced control development can be found in [35, 36]. Using dynamic inversion technique, the system can be divided into two main subsystems [37]. The first subsystem includes the internal dynamics, which are described by applying the feedback linearization technique and it is known by:

$$
\left[\begin{array}{c}
\ddot{x} \\
\ddot{y}
\end{array}\right]=\left[\begin{array}{c}
\theta \\
-\phi
\end{array}\right] \mathrm{g}
$$

Simple PD and PID controls are used to control the subsystems signal defined as:

$$
\begin{gathered}
P D\left(e_{i}\right)=P_{i} e_{i}+D_{i} \frac{d}{d t}\left(e_{i}\right) \\
P I D\left(e_{i}\right)=P_{i} e_{i}+I_{i} \int e_{i}+D_{i} \frac{d}{d t}\left(e_{i}\right)
\end{gathered}
$$

where $e_{i}$ represents the tracking error signal. For the first subsystem, two PD controllers are used to generate the command signal $\left[\phi_{c}, \theta_{c}\right]$. The tracking error is defined as:

$$
\left[\begin{array}{l}
e_{x} \\
e_{y}
\end{array}\right]=\left[\begin{array}{l}
x-x_{c} \\
y-y_{c}
\end{array}\right]
$$

Using the PID controller, the command signal could be found as:

$$
\begin{aligned}
& \phi_{c}=P D\left(e_{y}\right) \\
& \theta_{c}=P D\left(e_{x}\right)
\end{aligned}
$$

The fully actuated subsystem shown in equation 11 represents the second main subsystem and it is defined as:

$$
\left[\begin{array}{c}
\ddot{\phi} \\
\ddot{\theta} \\
\ddot{\psi} \\
\ddot{z}
\end{array}\right]=\left[\begin{array}{c}
1 / I_{x x} \\
1 / I_{y y} \\
1 / I_{z z} \\
\frac{1}{m}
\end{array}\right]\left[\begin{array}{c}
\tau_{\phi} \\
\tau_{\theta} \\
\tau_{\psi} \\
u_{z}
\end{array}\right]
$$

The altitude and attitude states $[z, \phi, \theta, \psi]$ are controlled using simple PD and PID controllers. On the other hand, the tracking error of the second subsystem is defined as:

$$
\left[\begin{array}{l}
e_{\phi} \\
e_{\theta} \\
e_{\psi} \\
e_{z}
\end{array}\right]=\left[\begin{array}{c}
\phi-\phi_{c} \\
\theta-\theta_{c} \\
\psi-\psi_{c} \\
z-z_{c}
\end{array}\right]
$$

The control signals for the second subsystem are defined as:

$$
\begin{gathered}
\tau_{\phi}=P D\left(e_{\phi}\right) \\
\tau_{\theta}=P D\left(e_{\theta}\right) \\
\tau_{\psi}=P I D\left(e_{\psi}\right) \\
u_{z}=P I D\left(e_{z}\right)+g
\end{gathered}
$$

The overall control scheme is illustrated in Figure 2. 


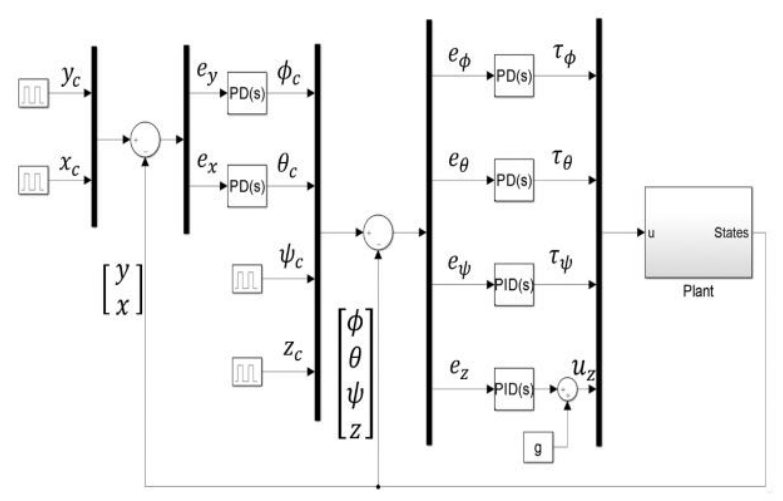

Figure 2: Quadrotor control scheme

\section{Optical flow sensor Model}

A special testing setup is designed to conduct the OF sensor's modeling experiment. The PX4FLOW kit is attached to the free-end of the pendulum, and a high accuracy encoder with 10,000 pulses per resolution (ppr) [38] is attached to the other end. This encoder measures the velocity of the pendulum while swaying. A distinguished landing pad with black stripes is placed underneath the OF sensor (see Figures 3 and 4). This allows the PX4FLOW sensor to recognize the moving features and obtain an accurate velocity measurement based on a fast image processing technique at $400 \mathrm{~Hz}$.

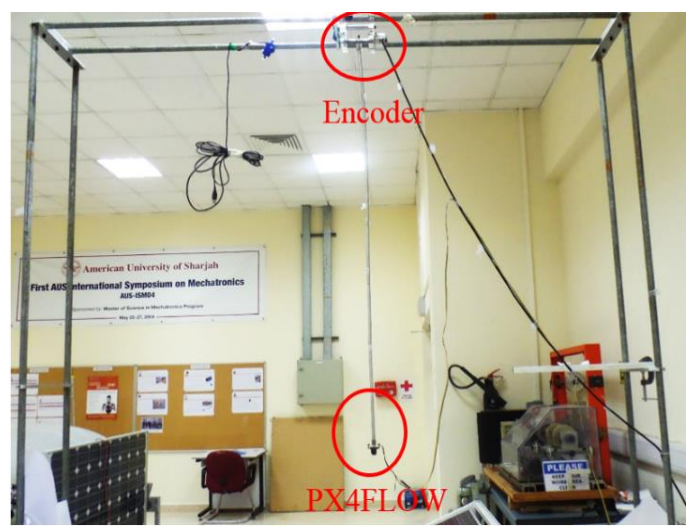

Figure 3. Optical flow sensor's pendulum test stand [39].

The optical flow readings are acquired via the serial port of the microcontroller. PX4FLOW data was acquired using the MAVlink protocol at 115200 baud rate [40] and transformed to the dSPACE system using another serial port. The encoder velocity and position measurements [38] were also read through the dSPACE system. By knowing these measurements, the translational velocities of the pendulum can be computed. The OF sensor's velocities along the $x$-axis and $y$-axis are measured and sent to a dSPACE unit utilizing the Real-Time Interface (RTI). The DS1104 R\&D Controller Board [41] was used to record the measurements of both sensors in real time.

A model for the PX4FLOW sensor was identified based on a system identification process. The system identification MATLAB toolbox (ident) was used to identify the dynamics of the optical flow sensor. The encoder measurements were used as the input to the OF model, and the OF sensor measurement was used as the output of the model, as shown in Figure 5.

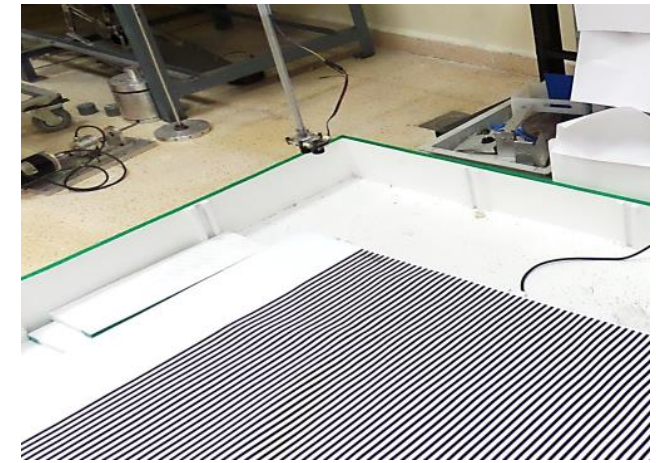

Figure 4. Landing pad [39]

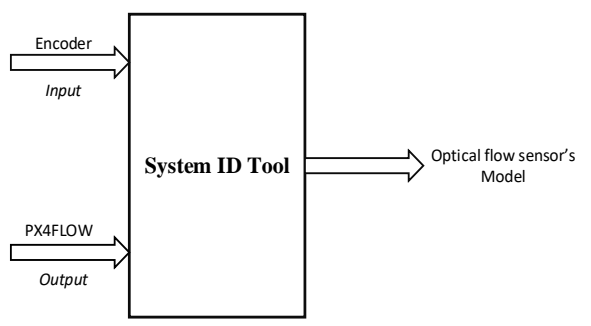

Figure 5. System identification scheme of OF model [39]

To obtain a precise model of the OF sensor that is robust to the change in the vehicle's altitude, the researchers have designed a pendulum with adjustable length to allow the $\mathrm{OF}$ sensor to be tested at several heights. The OF sensor was tested at four different heights $(60,80,100$ and $150 \mathrm{~cm})$ to characterize the variation of the accuracy with the change in altitude. The pendulum was displaced from its lowest height by a certain angle and the motion profile was recorded for both sensors subsequently.

The OF sensor dynamics are represented by a second order model which has a natural frequency of $13.85 \mathrm{rad} / \mathrm{sec}$ and a damping ratio of 0.53 [39]. This model was validated at the four different heights. This was done by inputting the encoder (truth) reading to the selected transfer function and comparing its output with the encoder's readings and the real PX4FLOW readings. As shown in Figure 6, the obtained OF model shows significant matching with the performance of the real PX4FLOW sensor.

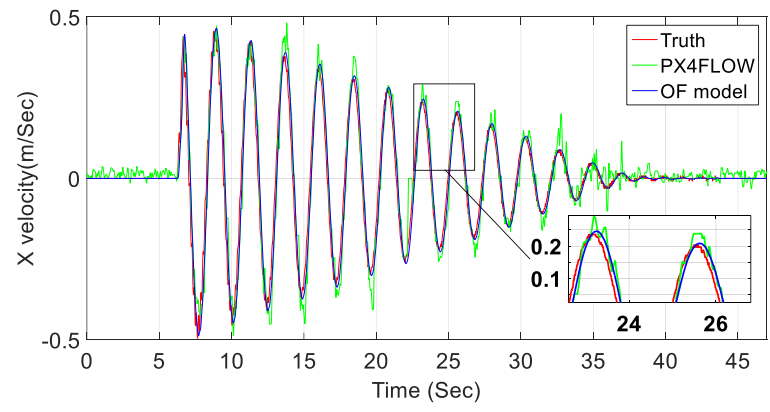

Figure 6: Validation test of the OF sensor's model.

As computed in Table 1, the selected model has small mean errors and small SD errors at various heights in comparison with the real PX4FLOW measurements. 
Table 1: Analytical comparison between the readings of the OF model and PX4FLOW at different heights.

\begin{tabular}{|c|c|c|}
\hline $\begin{array}{c}\text { Height } \\
\text { (cm) }\end{array}$ & $\begin{array}{l}\text { Mean } \\
\text { error } \\
(\mathrm{ME}) \\
(\mathbf{m} / \mathbf{s e c}) \\
\end{array}$ & $\begin{array}{c}\text { Standard } \\
\text { deviation } \\
\text { error } \\
\text { (SDE) }(\mathbf{m} / \mathbf{s e c}) \\
\end{array}$ \\
\hline 60 & 0.0064 & 0.0354 \\
\hline 80 & 0.0186 & 0.0642 \\
\hline 100 & 0.0613 & 0.1815 \\
\hline 150 & 0.0950 & 0.2815 \\
\hline
\end{tabular}

\section{MULTISENSOR DATA FUSION ARCHITECTURE}

The COTS GPS/INS unit provides position estimates with few meters error $[42,43]$. This estimation error is due to GPS/INS position measurement characteristics, such as the quality of the GPS receiver, multipath errors and the number of satellites in view. The resulting estimation error makes the GPS/INS insufficient as a stand-alone positional feedback system. Therefore, the precision landing is unguaranteed and an accurate augmentation for the GPS/INS measurements is needed. In [44], a precision landing with $27 \mathrm{~cm}$ maximum position error for the Maxi-Joker 3 helicopter was done using low cost GPS/INS/OF integrated solution. A PX4FLOW OF sensor was used to enhance the estimation accuracy of the GPS/INS system. In this study, the researchers assume that this algorithm is activated at the last few meters before touching the ground. Conventional and intelligent sensor fusion approaches have been implemented to obtain high-accuracy estimates of the dynamic states during the landing flight (see Figure 7). A model-based Kalman filter uses the attitude measurements of $\mathrm{IMU} \Psi_{m}=\left[\begin{array}{lll}\phi_{m} & \theta_{m} & \psi_{m}\end{array}\right]^{T}$ and $\Omega_{m}=\left[\begin{array}{lll}p_{m} & q_{m} & r_{m}\end{array}\right]^{T}$, 3axis body linear velocities $V_{m}=\left[\begin{array}{lll}u_{m} & v_{m} & w_{m}\end{array}\right]^{T}$ from the OF and the Altimeter readings $z_{m}$ to obtain an accurate estimate for the position, velocity and the attitude states while landing is being performed. Furthermore, an adaptive fuzzy sensor fusion is designed to adapt the measurement noise matrix $R$ through a well-tuned fuzzy inference system to obtain more accurate position estimates.

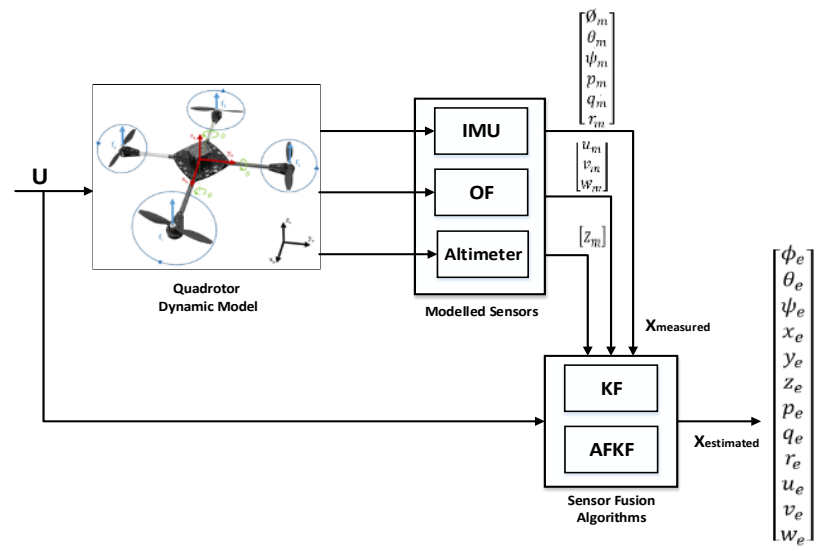

Figure 7: The sensor fusion scheme

\section{A. Kalman Filter design}

Considering the Gaussian assumption for the initial state error and all the noises associated with system model, the Kalman filter is considered an optimal Minimum Mean Square Error state estimator (MMSE) [20, 21, 16]. It provides an optimal estimation of the unknown states based on its recursive data processing algorithm. This estimation algorithm consists of the following two groups of equations:

1) State and measurement prediction equations

$$
\begin{aligned}
& \hat{x}_{k+1 \mid K}=A_{K} \hat{x}_{k \mid K}+B_{K} U_{K} \\
& P_{K+1 \mid k}=A_{K} P_{K \mid k} A_{K}^{T}+Q_{K}
\end{aligned}
$$

\section{2) State update equations}

$$
\begin{gathered}
v_{K+1}=z_{K+1}-H_{K+1} \hat{x}_{k+1 \mid K} \\
S_{K+1}=R_{K+1}+H_{K+1} P_{K+1 \mid k} H_{K+1}^{T} \\
W_{K+1}=P_{K+1 \mid k} H_{K+1}^{T} S_{K+1}^{-1} \\
\hat{x}_{k+1 \mid K+1}=\hat{x}_{k+1 \mid K}+W_{K+1} v_{K+1} \\
P_{K+1 \mid K+1}=P_{K+1 \mid k}-W_{K+1} S_{K+1} W_{K+1}^{T}
\end{gathered}
$$

Equations 16 and 17 update the current state and error covariance estimates from time step $K$ to $K+1$. These estimates are used as a priori estimates for the next cycle. Equation 16 is referred to as the innovation sequence which provides the filter by the additional information about the states in consequence to the new measurement $z_{K+1}$. Equations 16-18 combine the measurement updates into the a priori estimate to obtain and enhance the a posteriori estimate and estimate covariance (see equations 19 and 20).

Assuming that matrices $A_{K}, B_{K}, H_{K}, R_{K}$ and $Q_{K}$ noise matrices are known, the Kalman filter begins its state estimation cycle and a correction of the estimates is recursively performed as new measurements $z_{K}$ become available.

The design of such linear state estimator dictates linearizing the dynamic state space model of the quadrotor. The dynamic model has been linearized at hover where the Euler rates body velocities are around zero. As a result, the linear dynamic system is rewritten as follows:

$$
\left[\begin{array}{c}
\ddot{\phi} \\
\ddot{\theta} \\
\ddot{\psi} \\
\ddot{x} \\
\ddot{y} \\
\ddot{z}
\end{array}\right]=\left[\begin{array}{c}
\tau_{\phi} / I_{x x} \\
\tau_{\theta} / I_{y y} \\
\tau_{\psi} / I_{z z} \\
g \theta \\
-g \phi \\
u_{z} / m
\end{array}\right]
$$

The linear system is rewritten in state space form as:

$$
\begin{gathered}
\frac{d}{d t} x=A x+B u-G \\
y=C x
\end{gathered}
$$

where:

$$
\boldsymbol{x}=\left[\begin{array}{llllllllllll}
\phi & \theta & \psi & x & y & z & p & q & r & u & v & w
\end{array}\right]^{\mathrm{T}},
$$




$$
\begin{aligned}
& \mathbf{u}=\left[\begin{array}{llll}
\tau_{\phi} & \tau_{\theta} & \tau_{\psi} & \mathrm{u}_{\mathrm{z}}
\end{array}\right]^{\mathrm{T}} \\
& \mathbf{y}=\left[\begin{array}{llll}
x & y & \psi & z
\end{array}\right]^{\mathrm{T}} \\
& \mathbf{A}=\left[\begin{array}{cc}
\mathbf{0}_{6 \times 6} & \boldsymbol{I}_{6 \times 6} \\
a & \mathbf{0}_{10 \times 6}
\end{array}\right], \quad \mathrm{a}=\left[\begin{array}{cc}
0 & \frac{g}{m} \\
-\frac{g}{m} & 0 \\
0 & 0
\end{array}\right] \\
& B=\left[\begin{array}{c}
\mathbf{0}_{8 \times 4} \\
b
\end{array}\right] \\
& b=\left[\begin{array}{cccc}
1 / I_{x} & 0 & 0 & 0 \\
0 & 1 / I_{y} & 0 & 0 \\
0 & 0 & 1 / I_{z} & 0 \\
0 & 0 & 0 & 1 / m
\end{array}\right] \\
& G=\left[\begin{array}{c}
\mathbf{0}_{11 \times 1} \\
g
\end{array}\right] \\
& C=\left[\begin{array}{c}
\mathbf{0}_{8 \times 4} \\
0
\end{array}\right]
\end{aligned}
$$

The diagonal measurement noise covariance matrix $R_{\mathrm{k}}$ is given as:

$$
R_{k}=\left[\begin{array}{cccc}
r_{1,1} & 0 & \cdots & 0 \\
0 & r_{2,2} & & 0 \\
\vdots & & \ddots & \vdots \\
0 & 0 & \cdots & r_{9,9}
\end{array}\right]
$$

The diagonal process noise covariance matrix $Q_{\mathrm{k}}$ is also represented as:

$$
Q_{k}=\left[\begin{array}{cccc}
q_{1,1} & 0 & \cdots & 0 \\
0 & q_{2,2} & & 0 \\
\vdots & & \ddots & \vdots \\
0 & 0 & \ldots & q_{11,11}
\end{array}\right]
$$

The variances of $R_{\mathrm{k}}$ and $Q_{k}$ are chosen carefully while

\begin{tabular}{|c|c|}
\hline Measurements & Mean error (ME) \\
\hline \multicolumn{2}{|c|}{ Angular rates } \\
\hline Range & $\pm 300^{\circ} / \mathrm{s}$ \\
\hline Non-Linearity & $0.1 \%$ of FS \\
\hline Noise Density & $0.05^{\circ} / \mathrm{s} / \sqrt{H z}$ \\
\hline $3 \mathrm{~dB}$ Bandwidth & $20 \mathrm{~Hz}$ \\
\hline \multicolumn{2}{|c|}{ Acceleration } \\
\hline Range & $\pm 6 g$ \\
\hline Non-Linearity & $0.3 \%$ of FS \\
\hline Noise Density & $150 \mu \mathrm{g} / \sqrt{ } \mathrm{Hz}$ \\
\hline $3 \mathrm{~dB}$ Bandwidth & $20 \mathrm{~Hz}$ \\
\hline \multicolumn{2}{|c|}{$\begin{array}{c}\text { Attitude Accuracy (Tilt) } \\
0.4^{\circ}(1 \sigma)\end{array}$} \\
\hline \multicolumn{2}{|c|}{$\begin{array}{c}\text { Position Accuracy } \\
2 m C E P, W A A S / E G N O S\end{array}$} \\
\hline
\end{tabular}
designing the Kalman estimator. Knowing that the OF sensor is more precise than GPS/INS, the variances of the velocity states are chosen to be smaller than the variances of the position states.

A model of a low cost MIDG GPS/INS unit has been considered for obtaining the attitude and the body rates measurements at $50 \mathrm{~Hz}$ [45]. The measurements specifications of this unit are listed in Table 2.

Table 2: MIDG GPS/INS Measurements specifications.

\section{B. Adaptive Fuzzy Kalman fusion}

The adaptive estimation approach is based on recursive updating process for the diagonal elements of the statistical matrices $R_{\mathrm{k}}$ and/or $Q_{k}$. Assuming that the $Q_{k}$ matrix is totally known, an adaptive estimation to adapt $R_{\mathrm{k}}$ has been designed based on an innovation adaptive estimation (IAE) approach. This approach is based on a covariance-matching algorithm which investigates the consistency of the actual covariance of the residual with its theoretical value. As shown in equations 16-18, $S_{K+1}$ represents the theoretical covariance of the innovation sequence $v_{K+1}$. The actual covariance of the innovation sequence can be approximated using the following equation [20]:

$$
\hat{C}_{k+1}=(1 \backslash N) \sum_{i=i_{o}}^{N} v_{i} v_{i}^{T}
$$

where $N$ is the size of the estimation window and $i_{o}=k-N+$ 1 represents the first sample inside the estimation window. The window size has been chosen experimentally to be 50 , which gives a satisfactory smoothing of the statistical matrix.

As a covariance-matching approach is used in this study, a new variable has to be declared to represent the degree of matching between (DoM) $S_{K+1}$ and $v_{K+1}[20,21]$ as follows:

$$
D o M=S_{K+1}-\hat{C}_{k+1}
$$

After evaluating the discrepancy between the actual and the theoretical value of the covariance of the innovation sequence, a Fuzzy Inference System (FIS) has been tuned to compute the adjustment of the measurement noise matrix $R_{\mathrm{k}+1}$ at each state estimation cycle. According to equation 19, any change in $R_{\mathrm{k}}$ leads to a change in $S_{K+1}$ accordingly. Hence, the discrepancy between $S_{K+1}$ and $\hat{C}_{k+1}$ can be reduced by varying the value of $S_{K+1}$ through adjusting the value of $R_{\mathrm{k}+1}$. This nonlinear adjustment is governed by a set of IF-THEN fuzzy linguistic description rules as follows [21] :

1. If DoM is positive, this implies that $S_{K+1}$ is greater than $\hat{C}_{k+1}$; then decrease $R_{\mathrm{k}+1}$.

2. If DoM is negative, this implies that $S_{K+1}$ is smaller than $\hat{C}_{k+1}$; then increase $R_{\mathrm{k}+1}$.

3. If DoM is equal to zero (this implies that $S_{K+1}$ and $\hat{C}_{k+1}$ are equal; then maintain $R_{\mathrm{k}+1}$.

Hence, $R_{\mathrm{k}+1}$ can be adjusted as follows:

$$
R_{k+1}=R_{K}+\Delta R_{k+1}
$$

Where $\Delta R_{k+1}$ is the adaptation value which adjusts the $R_{k+1}$ at each estimation cycle. The proposed FIS takes the DoM value as an input and provides the adjustment value $\Delta R_{k+1}$ as an output. The Mamdani inference engine is used to provide the required $\Delta R_{k+1}$ from all the rules using min-max operators for composition, minimum operation for implication, as follows: 


$$
\mu_{\Delta R_{k+1}}=\bigcup\left(\mu_{D o M_{i}} \wedge \mu_{R_{k+1_{i}}}\right)
$$

where $\mu$ is the membership function used to represent the fuzzy linguistic terms for the input and output variables. Three different Gaussian bell-shaped membership functions are utilized to describe the DoM input variable linguistic terms: negative, zero and positive $\{\mathrm{N}, \mathrm{Z}, \mathrm{P}\}$, respectively, as shown in Figure 8. For the output variable $\Delta R_{k+1}$, shown in Figure 9, three different Gaussian bell-shaped membership functions are utilized to describe the variable linguistic terms as: decrease, maintain and increase $\{\mathrm{D}, \mathrm{M}, \mathrm{I}\}$, respectively.

A final crisp value for $\Delta R_{k+1}$ is fused from the accumulated output membership function from all the rules $\mu_{\Delta R}$. For this process, a centroid defuzzification function is used:

$$
\Delta R_{k+1}=\frac{\int \Delta R_{k+1} \mu\left(\Delta R_{k+1}\right) \mathrm{d} \Delta R}{\int \mu\left(\Delta R_{k+1}\right) \mathrm{d} \Delta R}
$$

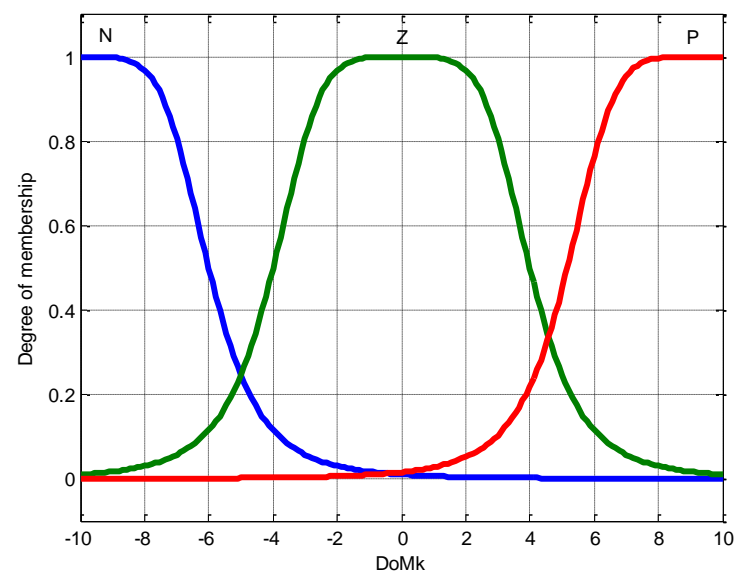

Figure 8: DoM linguistic terms

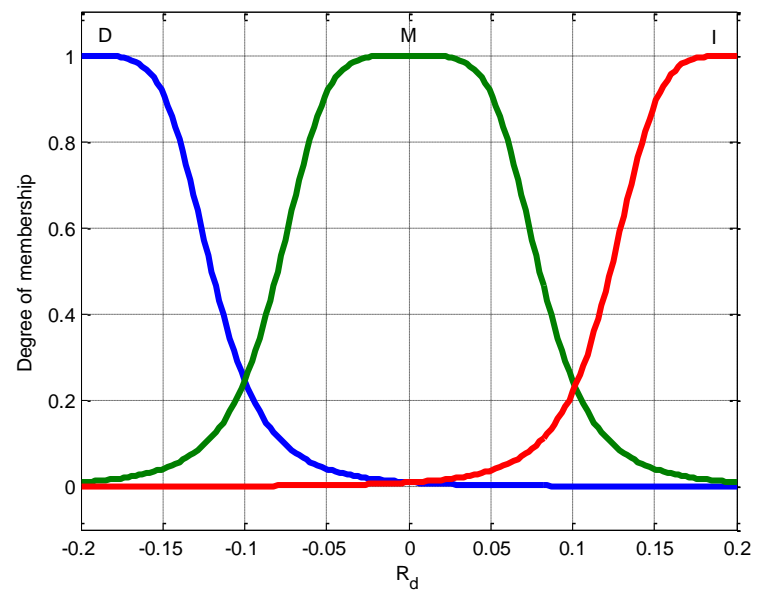

Figure 9: $\boldsymbol{\Delta} \boldsymbol{R}_{\boldsymbol{k}+1}$,linguistic terms

Figure 10 below illustrates the graphical representation of the Adapted fuzzy sensor fusion algorithm. In each Kalman cycle, each variance of the diagonal variances of the $\mathrm{R}$ matrix is adjusted separately.

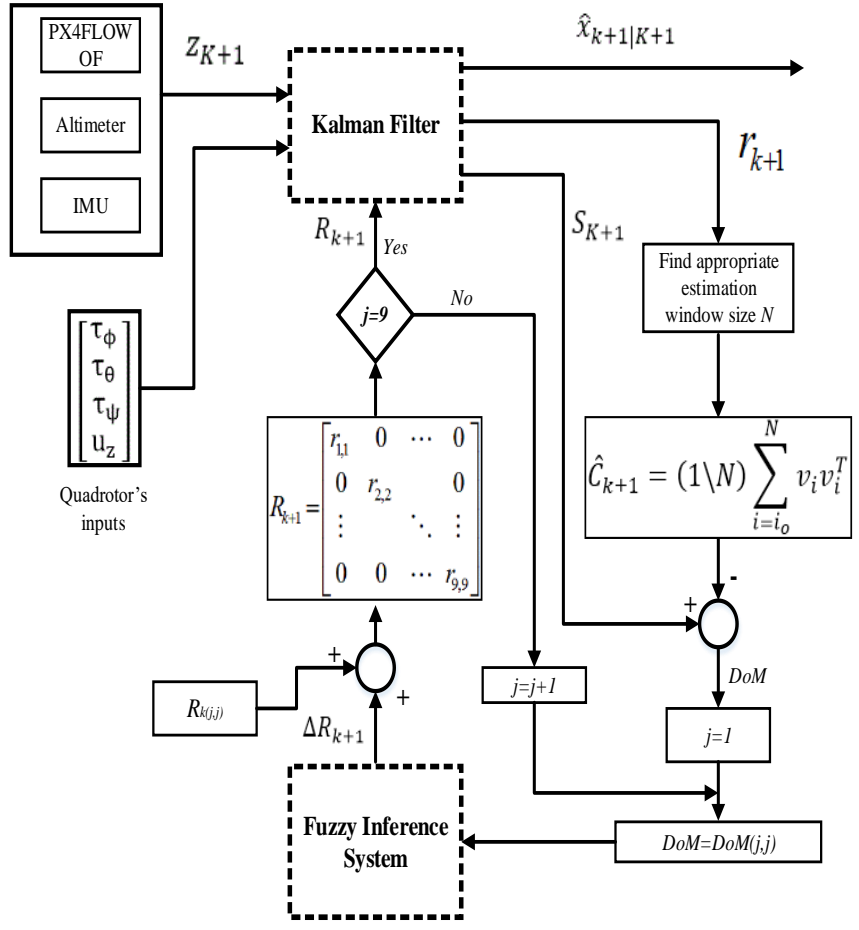

Figure 10: Graphical representation of AFKF algorithm

\section{Simulation Results}

A simulation environment was used to test the performance of the proposed sensor fusion method. The simulated data of real states were considered in controlling the landing process. In this section, the estimation results of the conventional and adaptive fuzzy sensor fusion approaches are presented. The presented approaches use the modeled measurements to estimate the attitude, velocity, altitude and the unknown position states of the quadrotor. A precision landing test was performed to validate the proposed estimation method. The velocity and the position are controlled and estimated in the quadrotor's body frame where the Z-axis is positive down (see Figure 1). In the landing test, the quadrotor is commanded to start descending from $-3 \mathrm{~m}$ altitude until it reaches the ground with a slope of $0.3 \mathrm{~m} / \mathrm{s}$. The quadrotor took approximately 10 seconds to land safely and precisely.

\section{A. Conventional state estimation}

The attitude, linear and angular velocities and the position states are estimated using a linear Kalman estimator in this section. The linear estimator shows a significant performance in estimating the quadrotor states while landing.

\section{1) Attitude estimation}

Figures 11 and 12 present the estimation of the attitude and the body rates states while landing is being performed. During the landing stage, the attitude angles are controlled to be around zero degrees to ensure safe landing. Figure 11 shows the real, measured and estimated attitude angles. It can be discerned that the proposed method estimates roll, pitch and yaw angles accurately. Figure 12 demonstrates the estimator's ability in 
terms of rejecting the associated measurement's noise and matching the real body rates of the quadrotor.
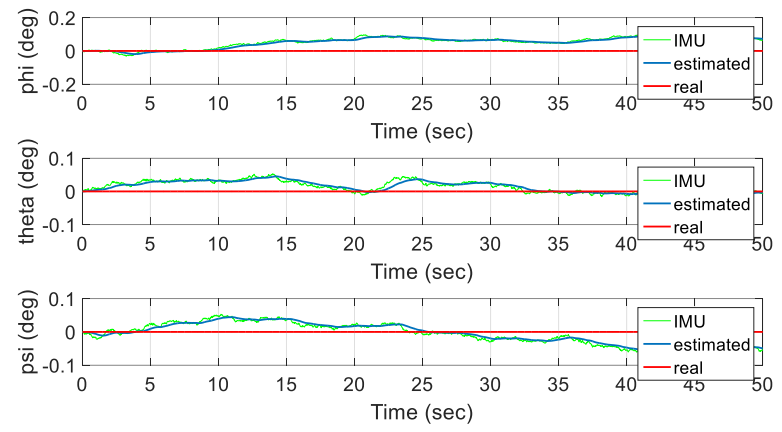

Figure 11: Attitude estimation.

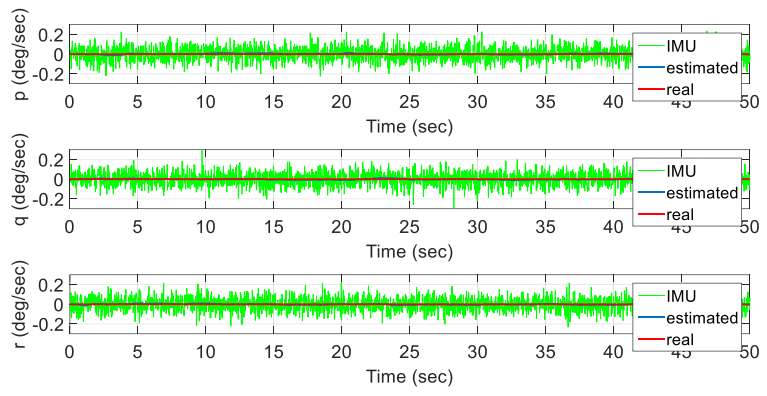

Figure 12: Angular body rates estimation

\section{2) Velocity estimation}

Figure 13 shows the estimated velocity profile during the landing stage. As the filter takes precise PX4FLOW measurements in $\mathrm{m} / \mathrm{sec}$ as velocity measurements, precise velocity estimates are expected.

The quadrotor performs slight velocity in $X$ and $Y$ while landing and these small velocities are estimated accurately as shown in Figure 13. As discerned, the measurement noise of the velocity has been significantly rejected.
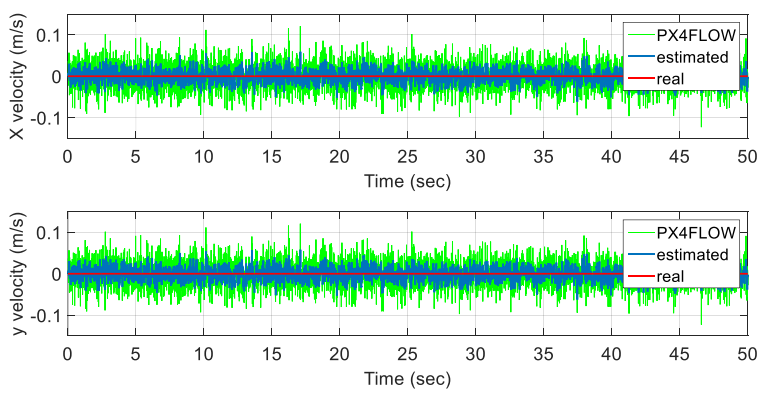

Figure 13: linear body velocities estimation

\section{3) Position estimation}

Figure 14 exhibits the state estimation of the unknown $X$ and $Y$ position states. From the Figure, it is clear that the $X$ position estimation converges to the real value of the state. On the other hand, the conventional KF has estimated $Y$ position with a maximum drift of $7.5 \mathrm{~mm}$. Similarly, as shown in Figure 15, the conventional estimator performs well in estimating the $Z$ position. The quadrotor is controlled to start descending from $3 \mathrm{~m}$ in $Z$ body frame until it reaches the ground with a slope of $0.3 \mathrm{~m} / \mathrm{s}$. The quadrotor took approximately 10 seconds to land safely and precisely (see the dashed red ellipse in Figure 15). The conventional Kalman estimator has succeeded in obtaining an accurate estimation of the altitude with $7 \mathrm{~cm}$ RMSE estimation error.
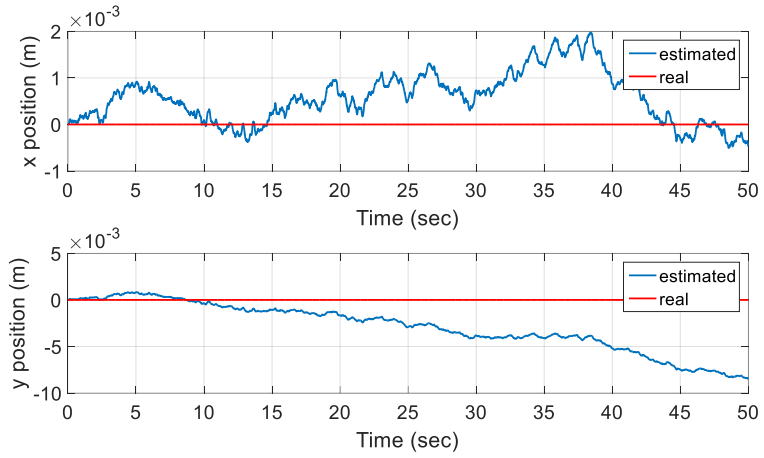

Figure 14: $\mathrm{X}$ and Y position estimation

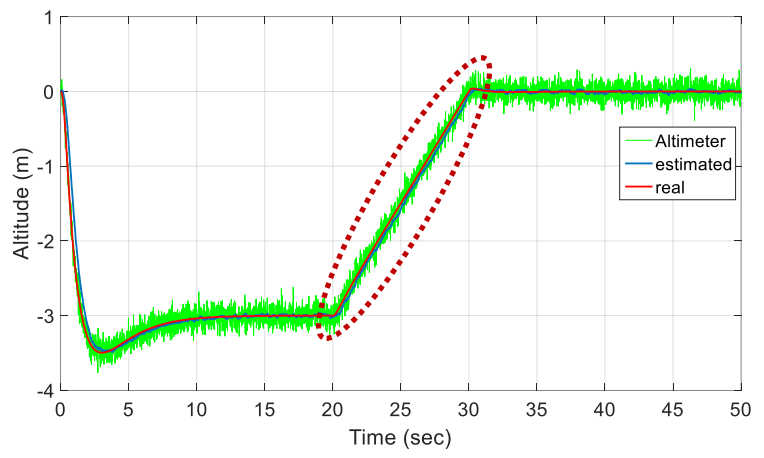

Figure 15: Altitude estimation

\section{B. Adaptive fuzzy state estimation}

The AFKF estimator has been developed in this study to obtain more accurate position state estimates while precision landing flight is being performed. The results of AFKF state estimation for the precision landing flight are presented in this section. Practically, the AFKF proves its superiority over the conventional filter in terms of estimating the unknown position states, body velocities and, most importantly, the altitude.

Figures 15 and 16 demonstrate the adaptation of the altitude measurement variance during landing. Figure 16 shows the adjustment amount $\Delta R_{k+1}$ of the altitude measurement variance; Figure 17 illustrates the adjusted $R$ variance for the altimeter measurements while landing. Figure 18 shows the adjusted $R$ variances for the PX4FLOW measurements.

Figure 19 presents the $\mathrm{X}$ and $\mathrm{Y}$ position states estimation during the landing phase. Both estimators have obtained accurate estimates of the $\mathrm{X}$ and $\mathrm{Y}$ axes. Knowing that no measurements are obtained for these states, from the figure, it can be observed that the AFKF still outperforms the normal KF in terms of having smaller divergence with the unmeasured real states. This means that the AFKF enhancement in estimating the other states has improved the estimation of the $\mathrm{X}$ and $\mathrm{Y}$ position states indirectly. Figure 20 shows that the AFKF estimated 
altitude has a significant matching with the real altitude. Statistically, the AFKF has obtained an altitude estimation with a Root Mean Square Estimation Error (RMSEE) of $0.04544 m$ while the $\mathrm{KF}$ has performed the altitude estimation with RMSEE of $0.06977 \mathrm{~m}$. This means that the AFKF has improved the altitude estimation by $34.87 \%$. Figure 21 presents the estimation errors of the position states.

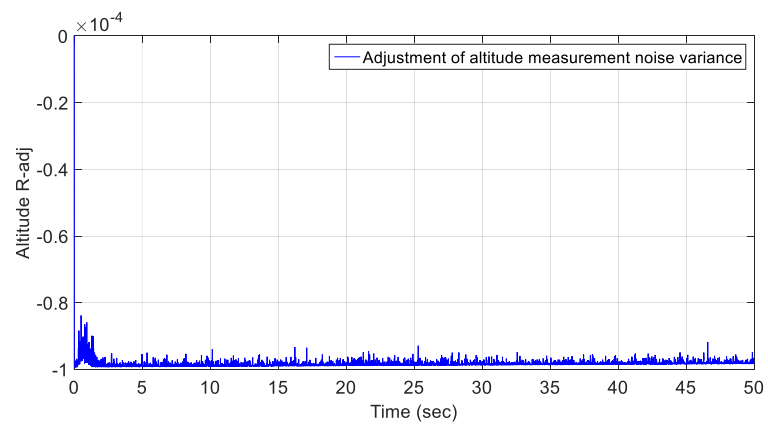

Figure 16: $R$ adjustment for the altitude measurement noise variance $\left(\Delta \boldsymbol{R}_{k+1}\right)$

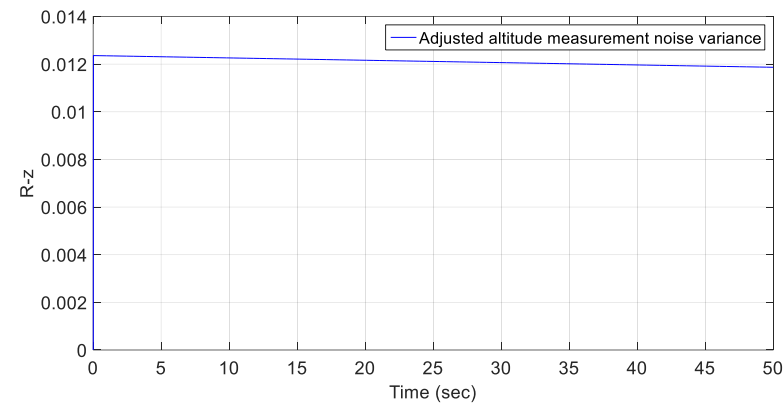

Figure 17: Adapted noise covariance for the altitude measurement $\left(\boldsymbol{R}_{\boldsymbol{k}+\mathbf{1}}\right)$
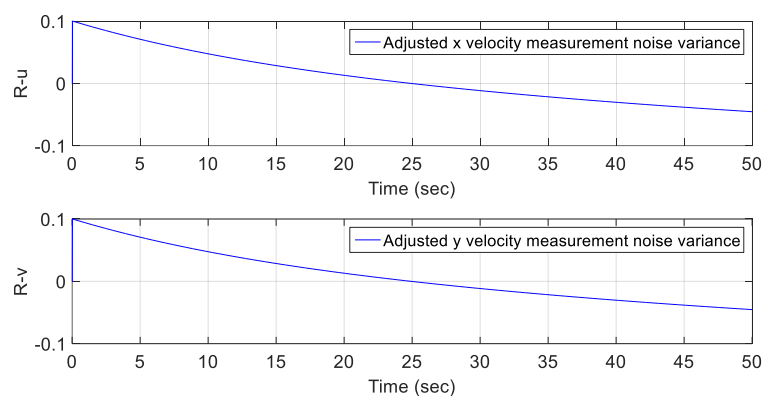

Figure 18: Adjusted variances of velocity noise measurements
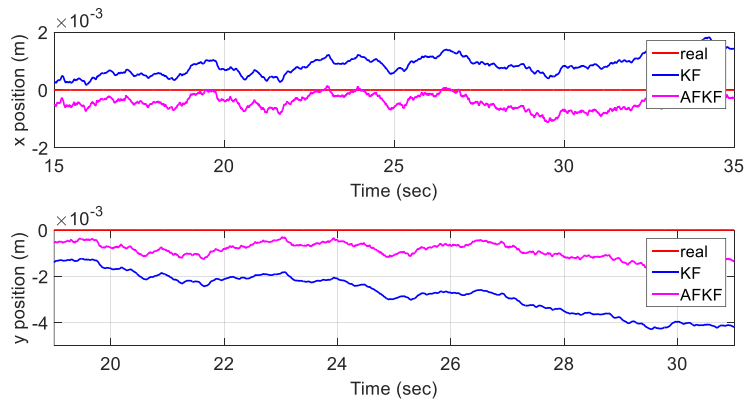

Figure 19: AFKF position estimation

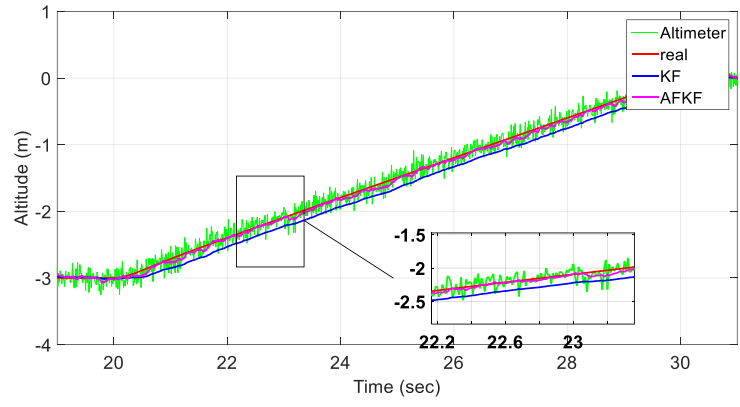

Figure 20: AFKF altitude estimate
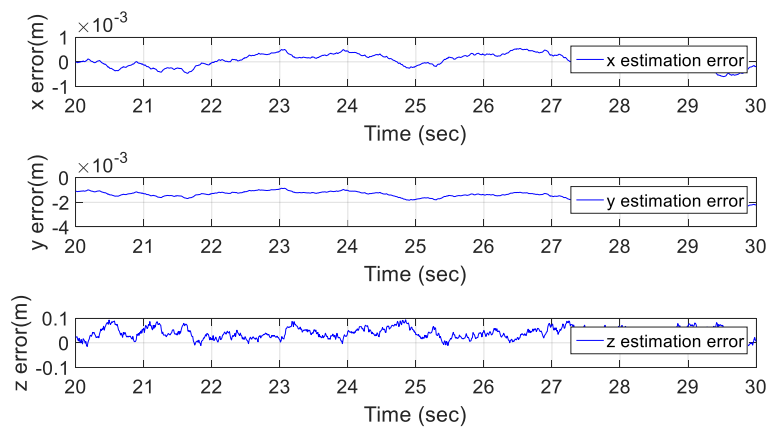

Figure 21: position estimation error during landing

The velocity states are estimated accurately as shown in Figure 22. $u$ and $v$ velocity states are estimated with less than 5 $\mathrm{cm} / \mathrm{sec}$ estimation error.

The used OF dynamic model was developed experimentally to include the dynamics of the sensor's accuracy and divergence [46]. Therefore, the OF divergence information was included in the obtained second order OF model. Figure 23 presents the vertical velocity estimation while landing. The quadrotor is descending at a constant speed of $0.3 \mathrm{~m} / \mathrm{s}$. As shown, AFKF has shown better performance in following the real state while the quadrotor is approaching the landing pad.
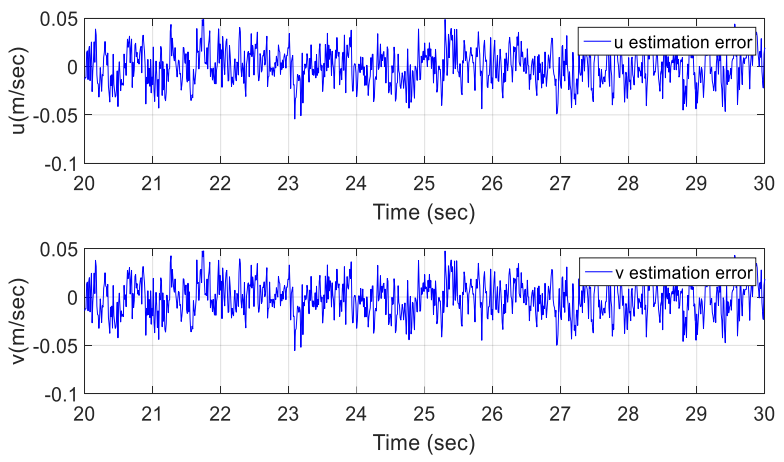

Figure 22: velocity estimation error during landing 


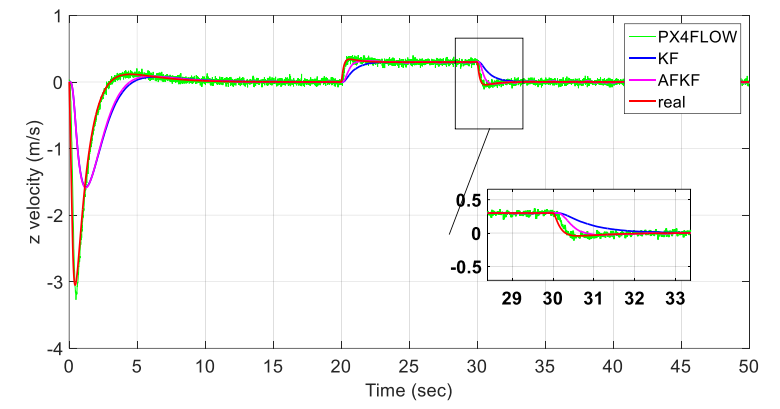

Figure 23: Vertical velocity estimation

\section{AFKF response to external disturbances}

This section investigates the dynamic response of the proposed intelligent estimator and its classical counterpart estimator while applying an external disturbance. A step disturbance of $5^{\circ}$ has been injected to the yaw state as shown in Figure 24. The AFKF estimator has shown superiority in terms of coinciding with the real state, convergence and settling time compared to the classical KF. The AFKF has improved the settling time of the KF by $23.3 \%$. From the Figure, it can be realized that the performance of the AFKF is significantly getting better as new measurements arrive. This confirms the accurate adaptation of the R matrix of the filter. In Figures 2526, noisy disturbances of $10^{\circ}$ and $20^{\circ}$ has been applied respectively to the heading state while landing. The AFKF has also shown a discerned ability of converging faster than the KF.

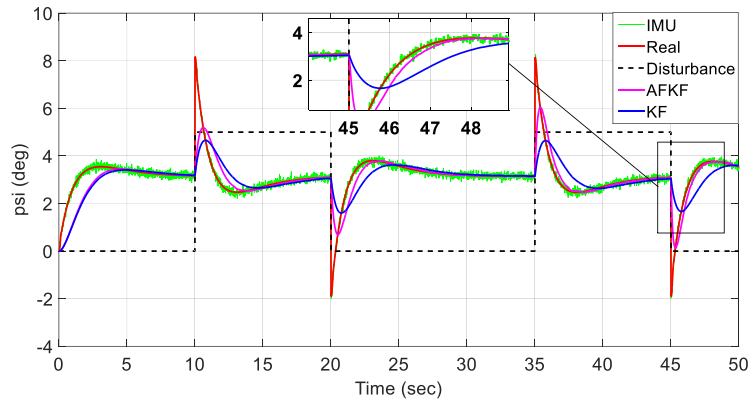

Figure 24: Estimators response a $5^{\circ}$ disturbance.

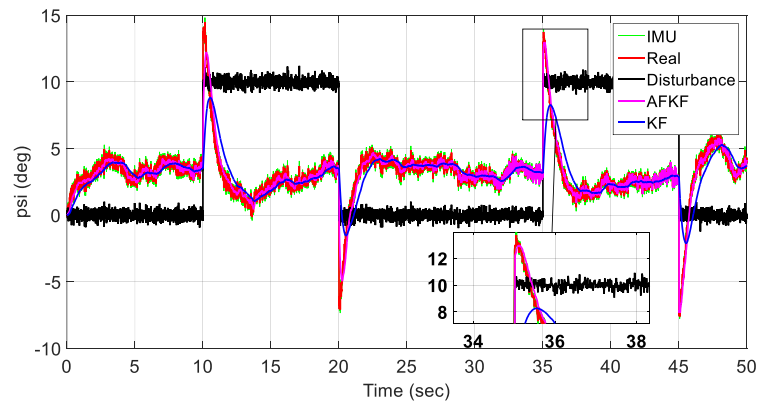

Figure 25: Estimators response to a $10^{\circ}$ disturbance

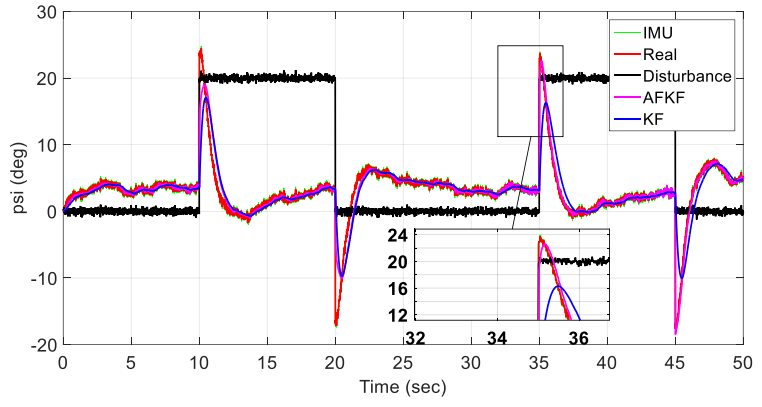

Figure 26: Estimators response to a $20^{\circ}$ disturbance.

\section{EXPERIENTAL VALIDATION}

In this section, a precision indoor landing experiment was conducted at different heights to validate the presented adaptive estimation technique. An F450 Quadrotor equipped with Pixhawk autopilot and PX4FLOW OF sensor was used for the precision landing experiment. Taking into account the Center of Gravity (CoG) of the quadrotor and the orientation of the Pixhawk autopilot, the PX4FLOW sensor was placed at the bottom of the quadrotor looking towards the landing pad (see Figures 27 and 28). The Pixhawk autopilot sampled the calibrated PX4FLOW readings through the $\mathrm{I}^{2} \mathrm{C}$ communication protocol at around $100 \mathrm{~Hz}$. The optimal resolution of the optical flow sensor was ensured through adjusting the parameters of the OF sensor using the QgroundControl software [15]. Moreover, a calibration process has been performed to ensure the clear view of the pattern by adjusting the lens position and monitoring the captured image by the PX4FLOW sensor using QGroundControl software. Using this software, the PX4FLOW velocities and vertical distance measurements were used for the velocity and the position control loops. A landing pad of $1.68 \mathrm{X} 1.18 \mathrm{~m}$ dimension and horizontal black stripes pattern was placed on the ground. The quadcopter was commanded to hover at several heights above the landing pad and land smoothly at approximately constant descending speed (see Figure 27).

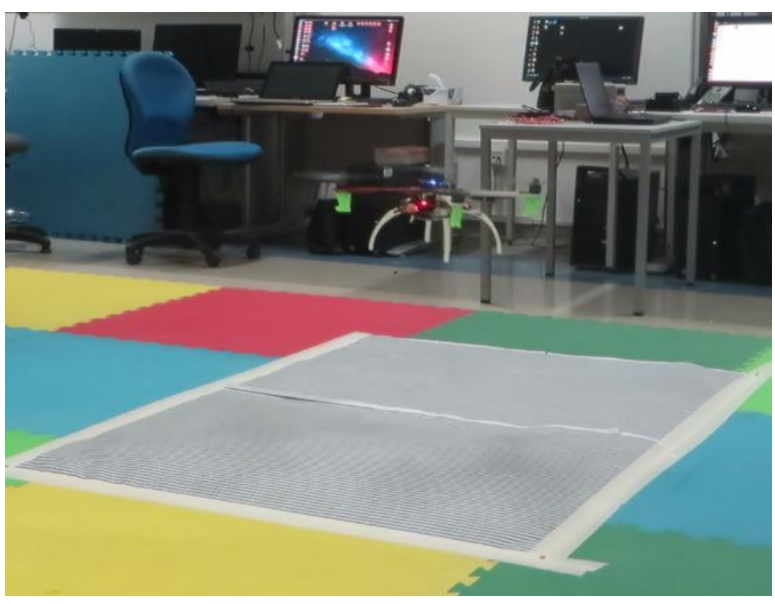

Figure 27: OF precision landing Experiment 


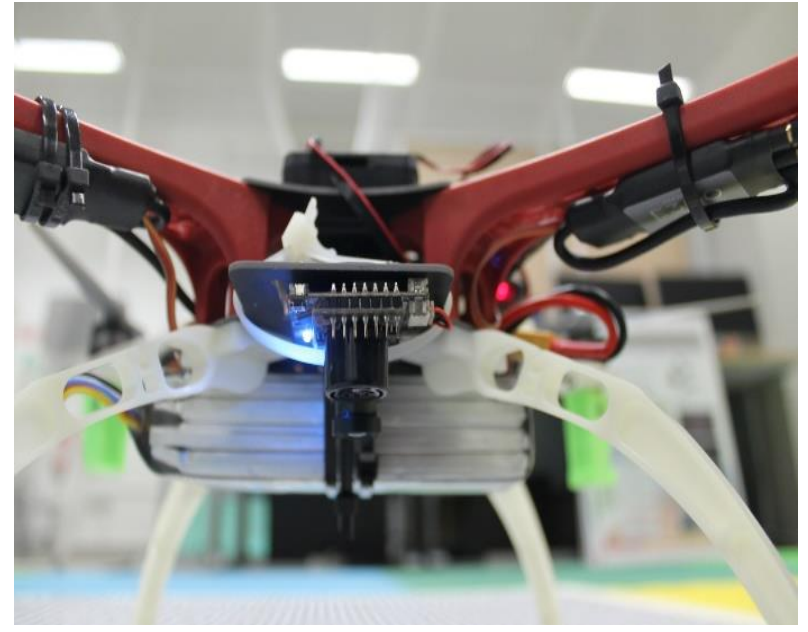

Figure 28: Mounted PXFLOW sensor

The section presents the proposed state estimation results while real precision landing is being performed at altitudes of $1 \mathrm{~m}, 1.5 \mathrm{~m}$ and $2 \mathrm{~m}$. The attitude, body velocities and position states are estimated using classical and adaptive state estimation techniques at each altitude.

\section{A. Descending from $1 \mathrm{~m}$}

In this section, the state estimation results of the attitude, body velocities and position states are illustrated while the Quadrotor is performing the precision landing at an altitude of $1 \mathrm{~m}$.

\section{1) Attitude estimation}

Figures 29-31 demonstrate the attitude estimation of the quadrotor while approaching the ground from an altitude of $1 \mathrm{~m}$. The performance of the proposed AFKF shows better coincidence with the measurement compared to the classical KF. The AFKF shows faster dynamic response and better convergence. It moves from its zero initial value and follows the measurement updates faster than the normal KF.

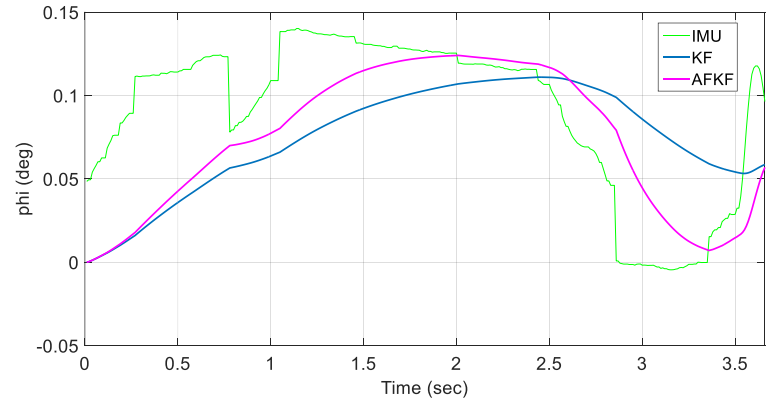

Figure 29: Roll state estimation

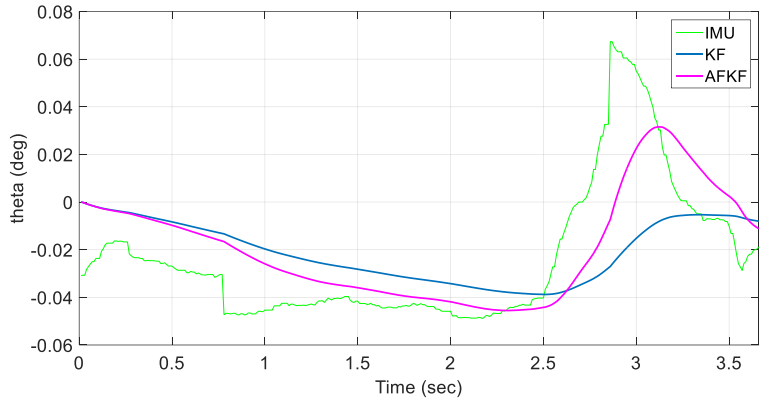

Figure 30: Pitch state estimation

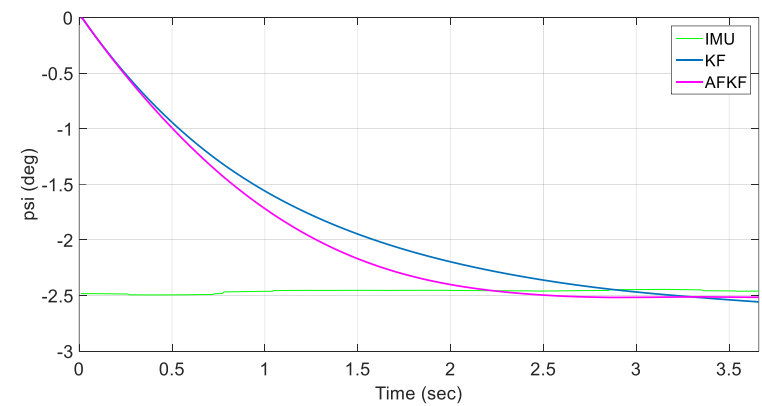

Figure 31: Heading state estimation

\section{2) Velocity estimation}

The results in Figures 32-34 exhibit the body velocities state estimation of the quadrotor while landing from a height of $1 \mathrm{~m}$. These states are measured by the PX4FLOW OF sensor. As shown, the AFKF has shown an agreement with the measured velocity states of the quadrotor.

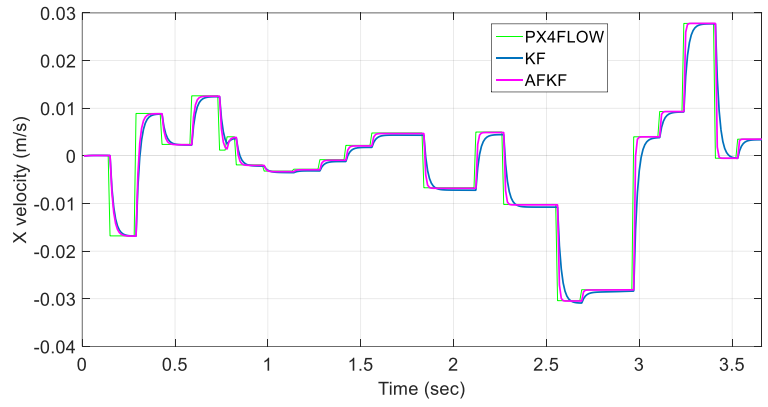

Figure 32: X velocity state estimation

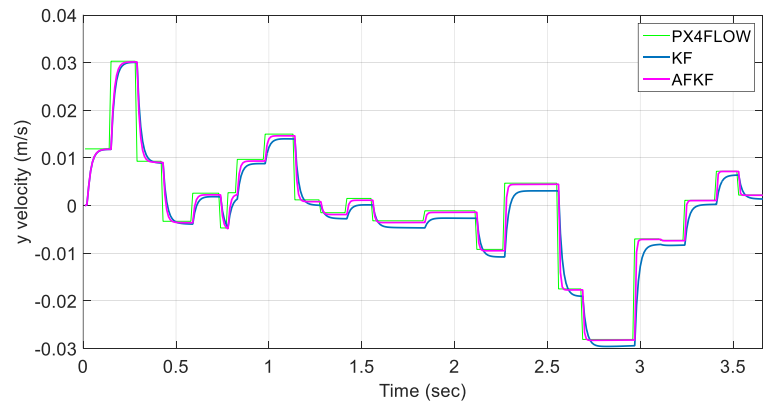

Figure 33: Y velocity state estimation 


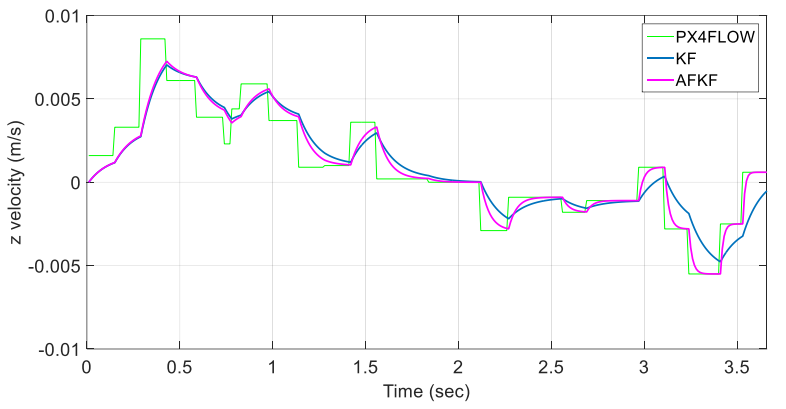

Figure 34: Z velocity state estimation

\section{3) Position estimation}

The quadrotor's position estimation is illustrated in Figures 35-37. From the Figures, it can be seen that both estimators has succeeded in obtaining estimates of the unmeasured slight motion in $\mathrm{X}$ and $\mathrm{Y}$ position states while precision landing is being carried out.

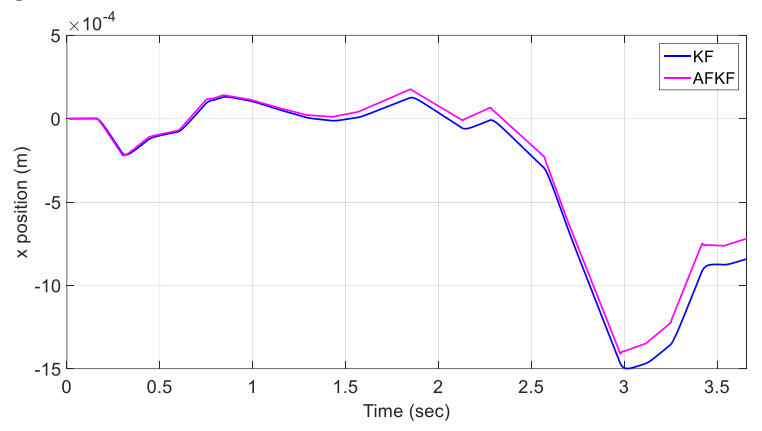

Figure 35: X position estimation

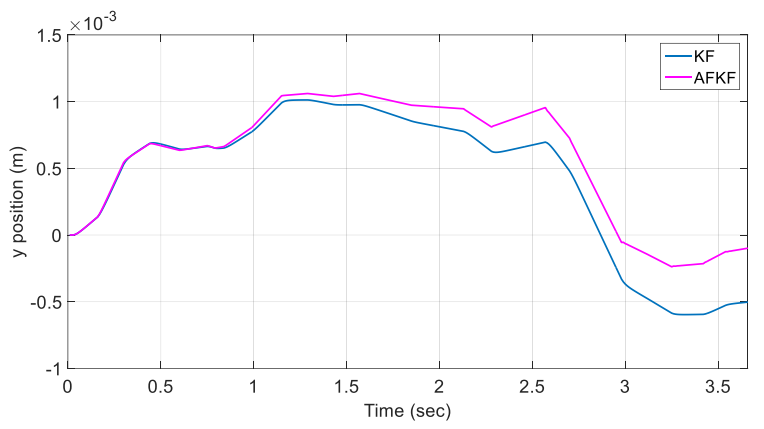

Figure 36: Y position estimation

Figure 40 shows the Altitude estimation of the quadrotor while descending. The measurements of the ultrasonic sensor of the PX4FLOW were considered for the altitude estimation. The AFKF has shown accurately adapted altitude estimation. It adapts its performance as the ultrasonic updates the altitude measurements.

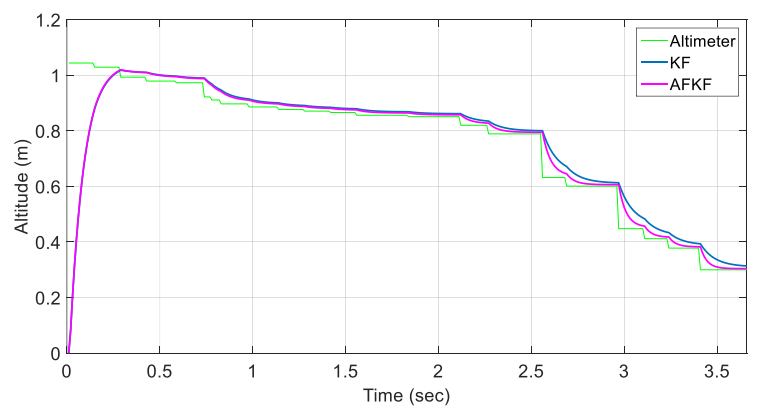

Figure 37: Altitude estimation

\section{B. Descending from $1.5 \mathrm{~m}$}

This section illustrate the state estimation of the attitude, body velocities and position states while descending from height of $1.5 \mathrm{~m}$.

\section{1) Attitude estimation}

Figures 38-40 show the attitude estimation of the quadrotor. Both estimators shows an agreement with the measurement of the roll, pitch and the heading states. The AFKF exhibits a faster dynamic response and better coincidence with the measured attitude changes of the vehicle while landing is being carried out.

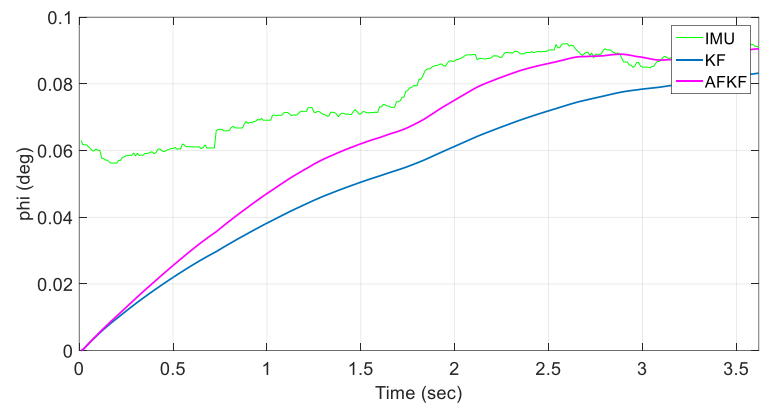

Figure 38: Roll state estimation

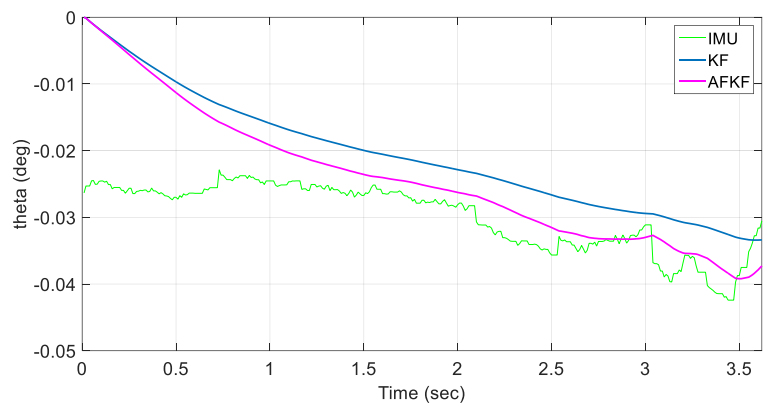

Figure 39: Pitch state estimation

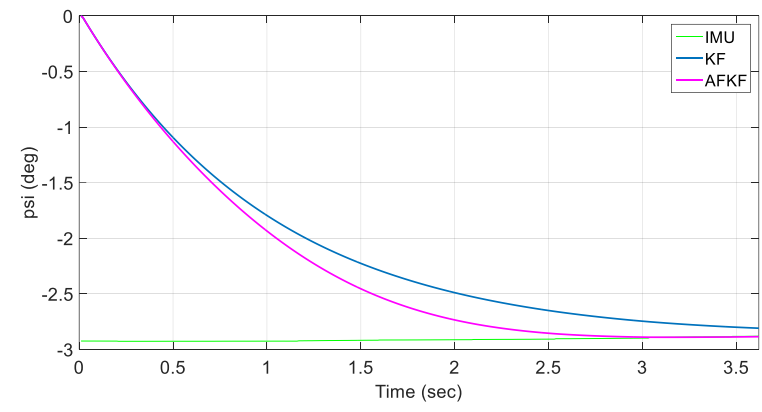

Figure 40: Heading state estimation

\section{2) Velocity estimation}

Figures 41-44 presents the body velocity states estimation of the vehicle while landing. According to these figures, the AFKF adapts its performance throughout the flight in order to ensure its agreement with the measured velocity states. 


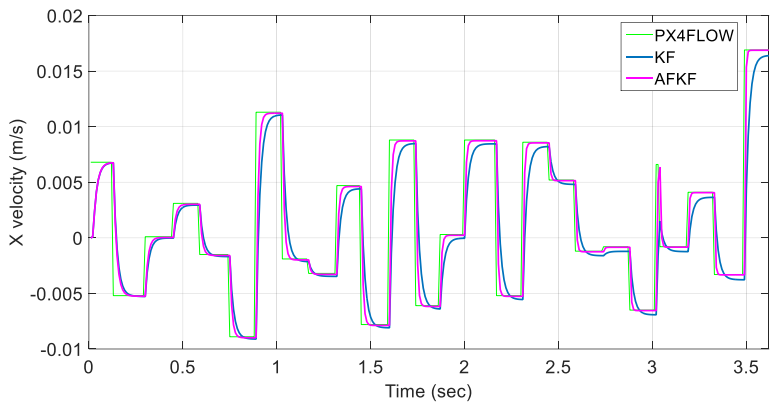

Figure 41: $\mathrm{X}$ body velocity estimation

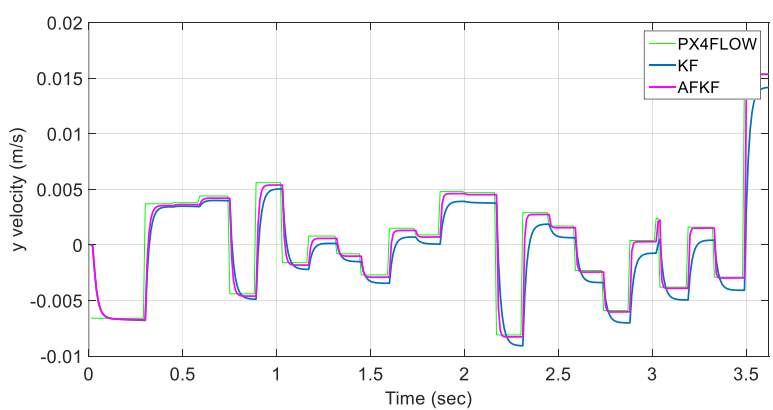

Figure 42: Y body velocity estimation

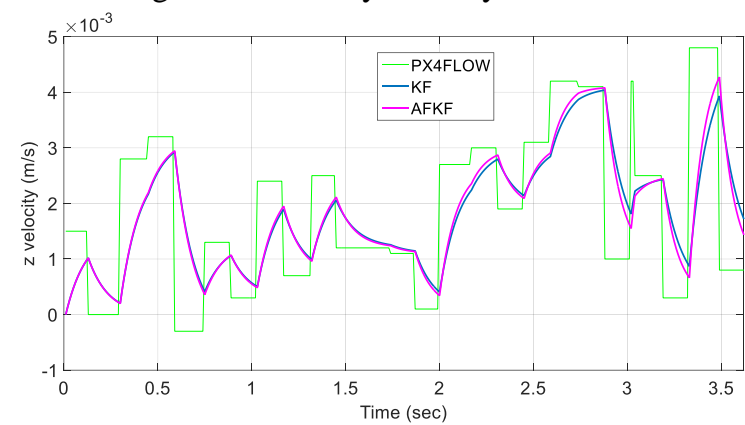

Figure 43: $\mathrm{Z}$ body velocity estimation

\section{3) Position estimation}

The position estimation is shown in Figures 44-46. The AFKF has estimated the slight motion in $\mathrm{X}$ and $\mathrm{Y}$ while landing from 1.5 is performed. Moreover, the AFKF has estimated the gradual descending of the vehicle. In figure 49, the AFKF shows better coincidence with ultrasonic measurement.

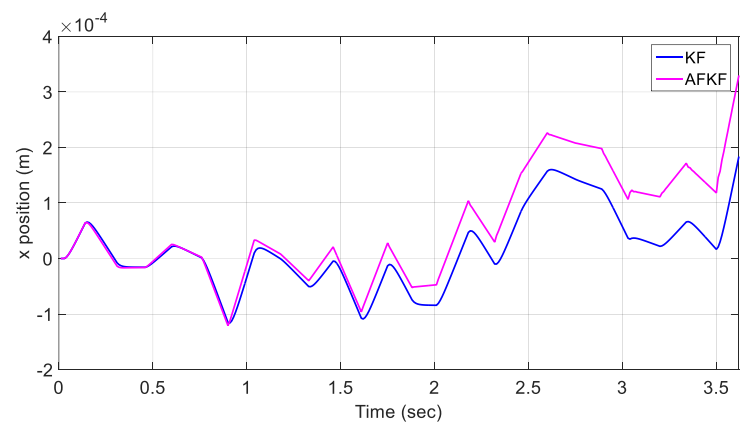

Figure 44: $\mathrm{X}$ position estimation

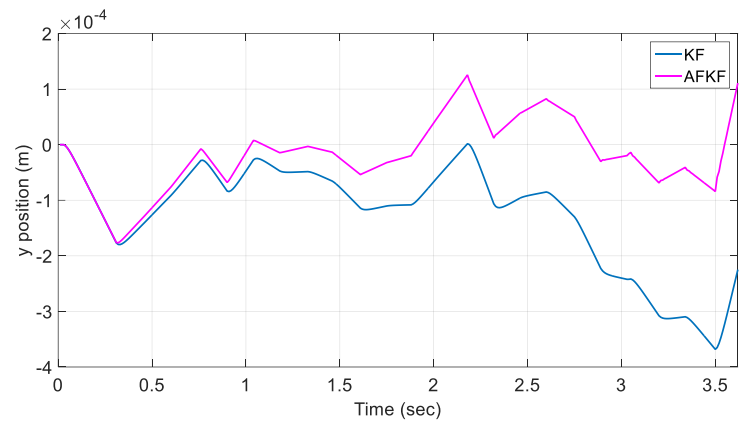

Figure 45: Y body position estimation

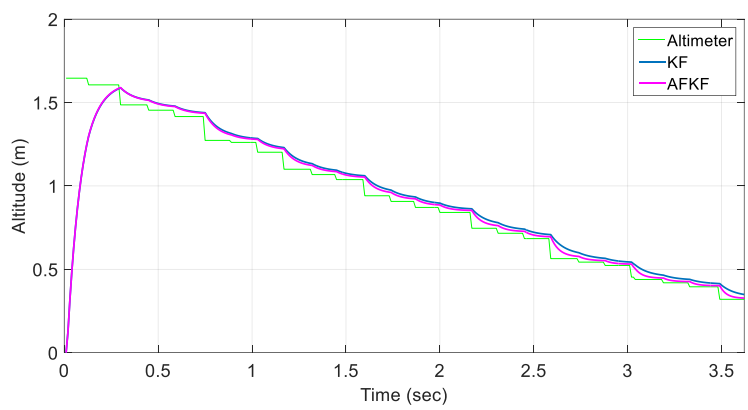

Figure 46: $\mathrm{Z}$ body position estimation

\section{Descending from $2 m$}

The section addresses the precision landing estimation results at higher height. The Quadrotor's state estimation has been performed while landing from $2 \mathrm{~m}$ height is being conducted.

\section{1) Attitude estimation}

Figures 47-49 represent the estimation of the quadrotor's attitude while landing from $2 \mathrm{~m}$. The AFKF has succeeded in following the measurement updates of the roll, pitch and heading states.

\section{2) Velocity estimation}

The velocity estimation is shown in Figures 50-52. The AFKF has estimated the small changes in the $\mathrm{X}$ and $\mathrm{Y}$ velocities while landing from $2 \mathrm{~m}$ is performed. As shown in figure 52, the AFKF has estimated the gradual descending of the vertical velocity of the vehicle.

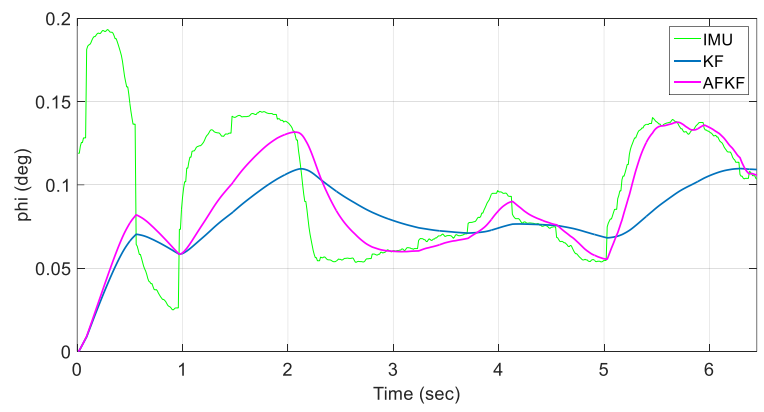

Figure 47: Roll state estimation 


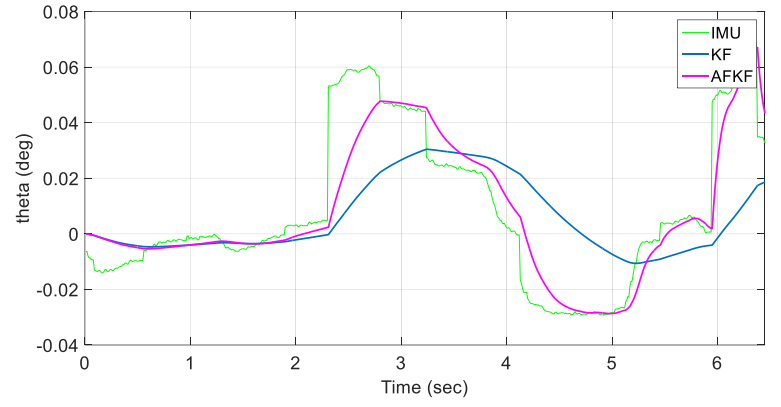

Figure 48: Pitch state estimation

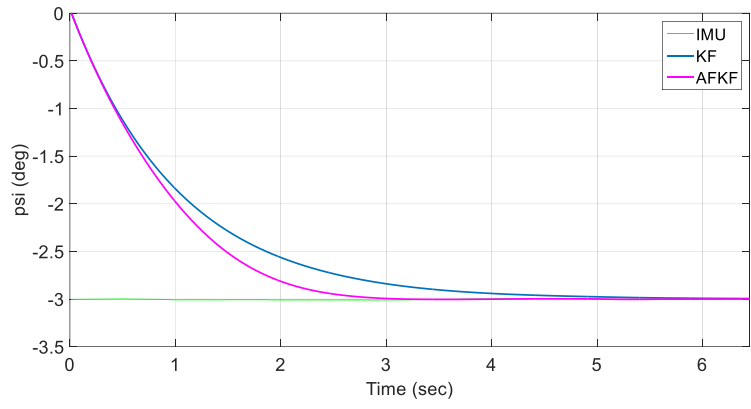

Figure 49: Heading state estimation

3) Position estimation

The position states are estimated as shown in Figures 53-55. The AFKF has estimated the small displacements in $\mathrm{X}$ and $\mathrm{Y}$ while landing from $2 \mathrm{~m}$ is performed. In Figure 55, the AFKF shows better coincidence with ultrasonic measurement.

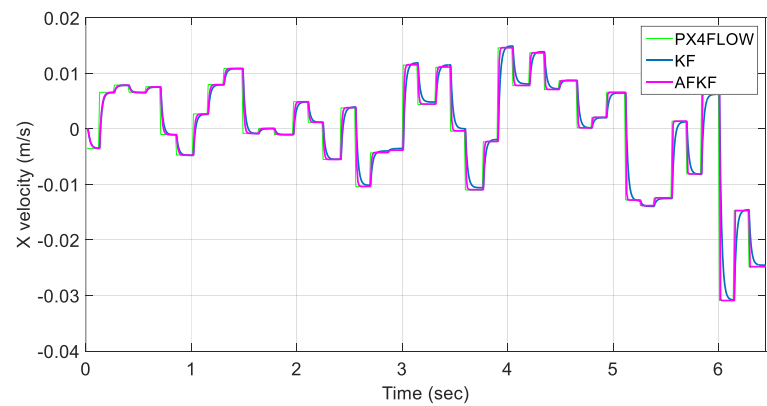

Figure 50: $\mathrm{X}$ body velocity estimation

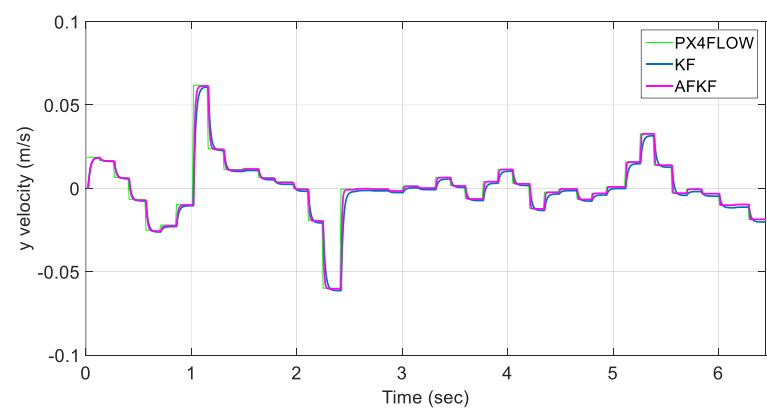

Figure 51: Y body velocity estimation

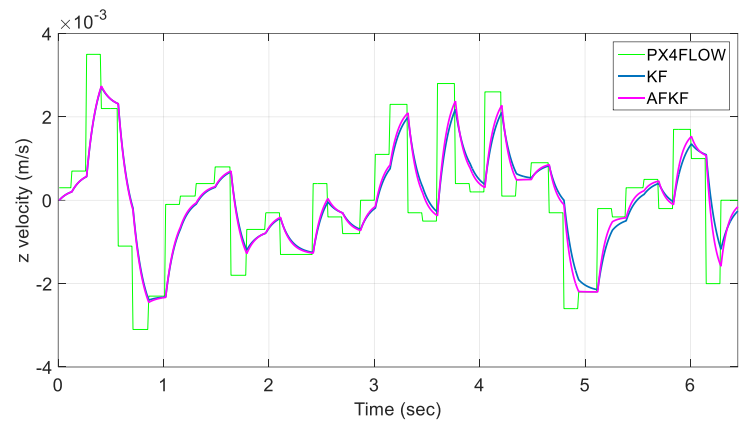

Figure 52: $\mathrm{Z}$ body velocity estimation

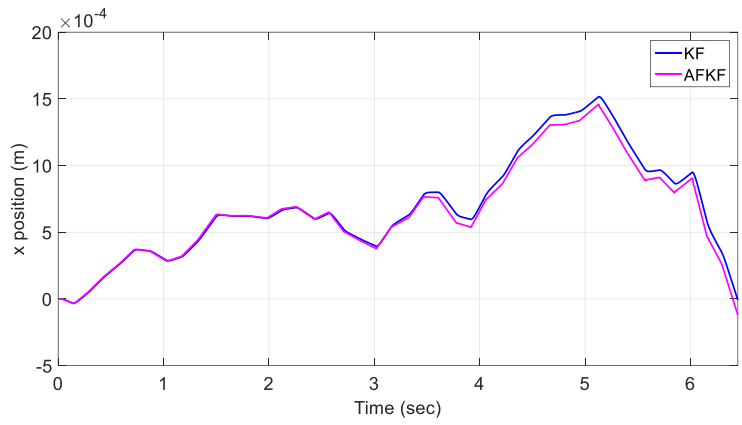

Figure 53: $\mathrm{X}$ body position estimation

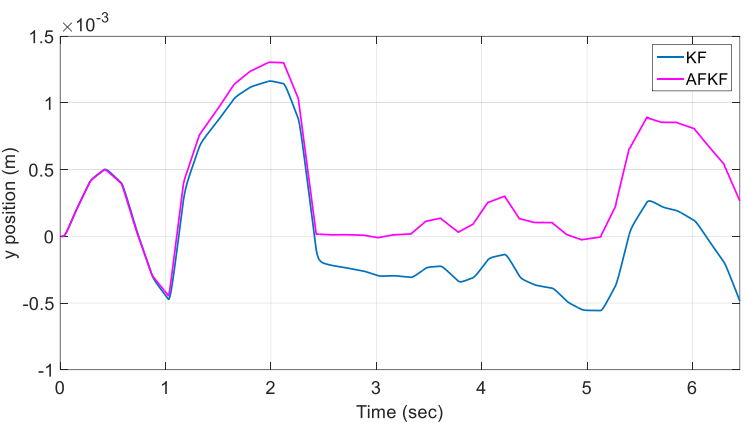

Figure 54: $\mathrm{Y}$ body position estimation

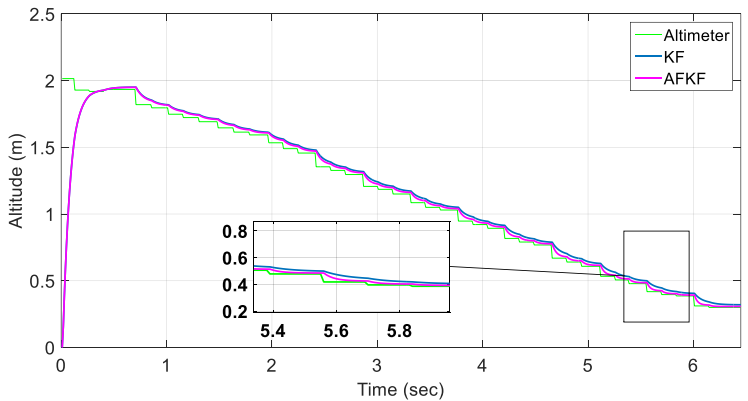

Figure 55: Z body position estimation

\section{CONCLUSION}

The problem of precise terminal landing phase of multi-rotor UAV's using low cost adaptive fuzzy multisensor data fusion architecture has been addressed in this paper. This low cost adaptive architecture can be used for vertical landings in areas where GPS outages might happen or in GPS denied areas. A model-based sensor fusion algorithm between the body linear velocities coming from the $\mathrm{OF}$ sensor, the attitude measurements of the INS unit and the altimeter readings is 
proposed. The conventional estimator has exhibited a rigorous performance in estimating the quadrotor states throughout the landing flights phases. For more precise states estimation and, more specifically, altitude estimation, an adaptive fuzzy data fusion algorithm has been developed. The presented AFKF results demonstrate the superiority of the Adaptive Fuzzy fusion technique in estimating the dynamic states over the conventional KF. The proposed AFKF shows an accurate adaptive altitude estimation and improves the estimation accuracy by $34.87 \%$ compared with the conventional KF. The accuracy of the obtained results allows the utilization of the proposed algorithm in the landing applications of quadrotor platforms in GPS-denied environments. As a future work of this research, a disturbance rejection capability along with a fault detection and recovery algorithm will be implemented to improve the accuracy of the proposed precision landing technique.

\section{REFERENCES}

[1] F. Jiancheng and Y. Sheng, "Study on Innovation Adaptive EKF for InFlight Alignment of Airborne POS," IEEE Transactions on Instrumentation and Measurement, vol. 60, no. 4, pp. 1378 - 1388, 2011.

[2] L. Chen and J. Fang, "A Hybrid Prediction Method for Bridging GPS Outages in High-Precision POS Application," IEEE Transactions on Instrumentation and Measurement, vol. 63, no. 6, pp. 1656 - 1665, 2014.

[3] B. J. Emran, M. Al-Omari, M. F. Abdel-Hafez and M. A. Jaradat, "A Cascaded Approach for Quadrotor's Attitude Estimation," Procedia Technology, vol. 15, pp. 268-277, 2014.

[4] B. Emran, M. Al-Omari, M. Abdel-Hafez and M. Jaradat, "Hybrid LowCost Approach for Quadrotor Attitude Estimation," ASME Journal of Computational and Nonlinear Dynamics, vol. 10, no. 3, pp. 1-9, 2015.

[5] F. Kendoul, I. Fantoni and K. Nonami, "Optic flow-based vision system for autonomous 3D localization and control of small aerial vehicles.," Robotics and Autonomous Systems, vol. 57, no. 6, pp. 591-602, 2009.

[6] G. Chowdhary, "GPS-denied Indoor and Outdoor Monocular Vision Aided Navigation and Control of Unmanned Aircraft," Journal of Field Robotics, vol. 30, no. 3, pp. 415-438, 2013.

[7] S. Saripalli, J. Montgomery and G. Sukhatme, "Visually-Guided Landing of an Unmanned Aerial Vehicle," IEEE Transactions on Robotics and Automation, vol. 19, no. 3, pp. 371-381, Jun 2003.

[8] K. E. Wenzel and A. Zell, "Automatic Take Off, Hovering and Landing Control for Miniature Helicopters with Low-Cost Onboard Hardware," in Proceedings of the AMS'09, Autonome Mobile Systeme, Karlsruhe, Germany, December 3-4 2009.

[9] C. Sharp, O. Shakernia and S. S. Sastry, "A Vision System for Landing an Unmanned Aerial Vehicle," in IEEE International Conference on Robotics and Automation, Seoul, Korea, 2001.

[10] S. Yang, S. Scherer and A. Zell, "An Onboard Monocular Vision System for Autonomous Takeoff,Hovering and Landing of a Micro Aerial Vehicle," Journal of Intelligent \& Robotic Systems, vol. 69, no. 4, pp. 499515, 2013.

[11] W. E. Green, P. Y. Oh, K. Sevcik and G. Barrows, "Autonomous Landing for Indoor Flying Robots Using Optic Flow," in Proceedings of IMECE'03 2003 ASME International Mechanical Engineering Congress, Washington, D.C., 2003.

[12] S. . R. Griffiths, "Remote Terrain Navigation for Unmanned Air Vehicles," in Master thesis, Brigham Young University, 2006.

[13] B. H'eriss'e, . T. Hamel, R. Mahony and . F.-X. Russotto, "Landing a VTOL Unmanned Aerial Vehicle on a Moving Platform Using Optical Flow," IEEE Transactions on Robotics, vol. 28, no. 1, pp. 77- 89, 2012.
[14] N. Gageik, M. Strohmeier and S. Montenegro, "An Autonomous UAV with an Optical Flow Sensor for Positioning and Navigation," International Journal of Advanced Robotic Systems, vol. 10, 2013.

[15] D. Honegger, . L. Meier, . P. Tanskanen and M. Pollefeys, "An Open Source and Open Hardware Embedded Metric Optical Flow CMOS Camera for Indoor and Outdoor Applications," in Robotics and Automation (ICRA), 2013 IEEE International Conference on. IEEE, 2013.

[16] M. Al-Sharman, "Attitude Estimation for a Small-Scale Flybarless Helicopter," in Multisensor Attitude Estimation: Fundamental Concepts and Applications, CRC Press, 2016, pp. 513-528 .

[17] D. A. Mercado, G. Flores, P. Castillo, J. Escareno and R. Lozano, "GPS/INS/Optic Flow Data Fusion for Position and Velocity estimation," in International Conference on Unmanned Aircraft Sysems (ICUAS), Atlanta,GA, 2013.

[18] M. Al-Sharman, M. . F. Abdel-Hafez and M. Al-Omari, "Attitude and Flapping Angles Estimation for aSmall-Scale Flybarless Helicopter Using a Kalman Filter," IEEE Sensors Journal, vol. 15, no. 4, pp. 2114 - 2122, 2015.

[19] M. Al-Sharman, M. Abdel-Hafez and M. Al-Omari, "State Estimation for a Small Scale Flybar-less Helicopter," in 2nd International Conference on System-Integrated Intelligence: Challenges for Product, Bremen, Germany, 2014

[20] A. H. Mohamed and K. P. Shwarz, "Adaptive Kalman Filtering for INS/GPS," Journal of Geodesy, vol. 73, no. 4, pp. 193-203, 1999.

[21] P. J. Escamilla-Ambrosio and N. Mort, "Development of a fuzzy logicbased adaptive Kalman filter," in Proceedings of the 2001 European Control Conference, Porto, Portugal , 2001.

[22] C.-M. Lin and C.-S. Hsueh, "Adaptive EKF-CMAC-Based Multisensor Data Fusion for Maneuvering Target," IEEE Transactions on Instrumentation and Measurement, vol. 62, no. 7, pp. 2058 - 2066, 2013.

[23] A. Makni, H. Fourati and A. Kibangou, "Energy-Aware Adaptive Attitude Estimation Under External Acceleration for Pedestrian Navigation," IEEE/ASME Transactions on Mechatronics, vol. 21, no. 3, pp. 1366 - 1375, 2015.

[24] K. Sebesta and N. Boizot, "A Real-Time Adaptive High-Gain EKF, Applied to a Quadcopter Inertial Navigation System," IEEE Transactions on Industrial Electronics, vol. 61, no. 1, pp. 495 - 503, 2013.

[25] D.-J. Jwo and T.-P. Weng, "An adaptive sensor fusion method with applications in integrated navigation," The Journal of Navigation, vol. 61, no. 4, pp. 705-721, 2008.

[26] H. Bian , J. Zhihua and T. Weifeng, "IAE-adaptive Kalman filter for INS/GPS integrated navigation system," Journal of Systems Engineering and Electronics, vol. 17, no. 3, pp. 502 - 508, 2006.

[27] C.-L. Lin, Y.-M. Chang, C.-C. Hung , C.-D. Tu and C.-Y. Chuang, "Position Estimation and Smooth Tracking With a Fuzzy-Logic-Based Adaptive Strong Tracking Kalman Filter for Capacitive Touch Panels," IEEE Transactions on Industrial Electronics, vol. 62, no. 8, pp. 5097 $5108,2015$.

[28] W. Abdel-Hamid, . A. Noureldin and . N. El-Sheimy, "Adaptive Fuzzy Prediction of Low-Cost Inertial-Based Positioning Errors," IEEE Transactions on Fuzzy Systems, vol. 15, no. 3, pp. 519 - 529, 2007.

[29] D.-J. Jwo and S.-H. Wang, "Adaptive Fuzzy Strong Tracking Extended Kalman Filtering for GPS Navigation," IEEE Sensors Journal, vol. 7, no. 5, pp. 778 - 789, 2007.

[30] K. Kanistras, G. Martins, . M. J. Rutherford and K. P. Valavanis, "A survey of unmanned aerial vehicles (UAVs) for traffic monitoring," in 2013 International Conference on Unmanned Aircraft Systems (ICUAS), Atlanta, GA, 2013.

[31] E. G. James , Matrix Algebra Theory, Computations, and Applications in Statistics, springer, 2007.

[32] R. Mahony , V. Kumar and P. Corke , "Multirotor Aerial Vehicles: Modeling, Estimation, And Control of Quadrotor," IEEE Robotics \& Automation Magazine, vol. 19, no. 3, pp. 20-32, 2012. 
[33] M.-D. Hua, . T. Hamel , P. Morin and C. Samson, "Introduction to feedback control of underactuated VTOLvehicles: A review of basic control design ideas and principles," IEEE Control Systems, vol. 33, no. 1, pp. 61-75, 2013.

[34] Y. Li and S. Song, "A survey of control algorithms for Quadrotor Unmanned Helicopter," in 2012 IEEE Fifth International Conference on Advanced Computational Intelligence (ICACI), Nanjing, 2012.

[35] A. Yesildirek and B. Imran, "Nonlinear control of quadrotor using multi Lyapunov functions," in American Control Conference, 2014.

[36] B. Emran and A. Yesildirek, "Robust nonlinear composite adaptive control of quadrotor," International Journal of Digital Information and Wireless Communications, vol. 4, no. 2, pp. 213-225, 2014.

[37] A. Das, F. L. Lewis and K. Subbarao, "Sliding Mode Approach to Control Quadrotor Using Dynamic Inversion," in Challenges and Paradigms in Applied, RijeKa, InTech, 2011.

[38] "Baumer encoders," Baumer, August 2016. [Online]. Available: http://www.baumer.com/us-en/products/rotary-encoders-anglemeasurement/absolute-encoders/. [Accessed August 2016].

[39] M. Al-Sharman, M. A. Al-Jarrah and M. Abdel-Hafez, "Auto Takeoff and Precision Terminal-Phase Landing using an Experimental Optical Flow Model for GPS/INS Enhancement," ASCE-ASME Journal of Risk and Uncertainty in Engineering Systems, Part B: Mechanical Engineering, 2018.

[40] "PX4FLOW Smart Camera," 2014. [Online]. Available: https://pixhawk.org/modules/px4flow.

[41] "DS1104 R\&D Controller Board," dspace GmpH, August 2016. [Online]. Available:

http://www.dspace.com/en/pub/home/products/hw/singbord/ds1104.cfm. [Accessed August 2016].

[42] J. Fang and X. Gong, "Predictive Iterated Kalman Filter for INS/GPS Integration and Its Application to SAR Motion Compensation," IEEE Transactions on Instrumentation and Measurement, vol. 59, no. 4, pp. 909 $-915,2010$.

[43] X. Gong, J. Zhang and J. Fang, "A Modified Nonlinear Two-Filter Smoothing for High-Precision Airborne Integrated GPS and Inertial Navigation," IEEE Transactions on Instrumentation and Measurement, vol. 64, no. 12, pp. 3315 - 3322, 2015.

[44] M. Al-Sharman, "Auto Takeoff and Precision Landing using Integrated GPS/INS/Optical Flow Solution," American University of Sharjah, Shajah, UAE, 2015.

[45] "Omni Instruments,", 2013. [Online]. Available: http://www.omniinstruments.co.uk/gyro/MIDGII.htm.

[46] H. W. Ho, G. C. d. Croon and Q. Chu, "Distance and velocity estimation using optical flow from a monocular camera," International Journal of Micro Air Vehicles , vol. 9, no. 3, pp. 198-208, 2017. 